\title{
Neuroprotective properties of queen bee acid by autophagy induction
}

\author{
Guadalupe Martínez-Chacón (iD) - Marta Paredes-Barquero (iD) • \\ Sokhna M.S Yakhine-Diop • Elisabet Uribe-Carretero • Ariadna Bargiela • \\ María Sabater-Arcis • José Morales-García • Jesús Alarcón-Gil • Eva Alegre-Cortés • \\ Saray Canales-Cortés • Mario Rodríguez-Arribas • Pedro Javier Camello • \\ José Manuel Bravo-San Pedro • Ana Perez-Castillo • Rubén Artero • \\ Rosa A. Gonzalez-Polo (D) • José M. Fuentes $(\mathbb{D}) \cdot$ Mireia Niso-Santano
}

Received: 15 January 2021 / Accepted: 21 June 2021

(C) The Author(s) 2021

\begin{abstract}
Autophagy is a conserved intracellular catabolic pathway that removes cytoplasmic components to contribute to neuronal homeostasis. Accumulating evidence has increasingly shown that the induction of autophagy
\end{abstract}

Guadalupe Martínez-Chacón and Marta Paredes-Barquero are authors of equal contribution.

Rosa A. Gonzalez-Polo, José M. Fuentes, and Mireia Niso-

Santano are authors of equal contribution.

G. Martínez-Chacón • M. Paredes-Barquero •

S. M. Yakhine-Diop • E. Uribe-Carretero •

E. Alegre-Cortés · S. Canales-Cortés ·

M. Rodríguez-Arribas · R. A. Gonzalez-Polo •

J. M. Fuentes · M. Niso-Santano

Departamento de Bioquímica y Biología Molecular y Genética, Facultad de Enfermería y Terapia Ocupacional, Universidad de Extremadura, Avda de la Universidad s/n, 10003 Cáceres, Spain

G. Martínez-Chacón

e-mail: guadalupemc@unex.es

M. Paredes-Barquero

e-mail: martapb@unex.es

S. M. Yakhine-Diop

e-mail: smsyakhinediop@unex.es

E. Uribe-Carretero

e-mail: euribec@unex.es

E. Alegre-Cortés

e-mail: ealegrec@unex.es improves neuronal health and extends longevity in several animal models. Therefore, there is a great interest in the identification of effective autophagy enhancers with potential nutraceutical or pharmaceutical properties to ameliorate
G. Martínez-Chacón · S. M. Yakhine-Diop •

E. Uribe-Carretero · J. Morales-García •

M. Rodríguez-Arribas · J. M. B.-S. Pedro •

A. Perez-Castillo • R. A. Gonzalez-Polo • J. M. Fuentes • M. Niso-Santano

Centro de Investigación Biomédica en Red de Enfermedades Neurodegenerativas (CIBERNED), Madrid, Spain

J. Morales-García

e-mail: jmorales@iib.uam.es

J. M. B.-S. Pedro

e-mail: josemabr@ucm.es

A. Perez-Castillo e-mail: aperez@iib.uam.es 
age-related diseases, such as neurodegenerative disorders, and/or promote longevity. Queen bee acid (QBA, 10hydroxy-2-decenoic acid) is the major fatty acid component of, and is found exclusively in, royal jelly, which has beneficial properties for human health. It is reported that QBA has antitumor, anti-inflammatory, and antibacterial activities and promotes neurogenesis and neuronal health; however, the mechanism by which QBA exerts these effects has not been fully elucidated. The present study investigated the role of the autophagic process in the protective effect of QBA. We found that QBA is a novel autophagy inducer that triggers autophagy in various neuronal cell lines and mouse and fly models. The beclin-1 (BECN1) and mTOR pathways participate in the regulation of QBA-induced autophagy. Moreover, our results showed that QBA stimulates sirtuin 1 (SIRT1), which promotes autophagy by the deacetylation of critical ATG proteins. Finally, QBA-mediated autophagy promotes neuroprotection in Parkinson's disease in vitro and in a mouse model and extends the lifespan of Drosophila melanogaster. This study provides detailed evidences

G. Martínez-Chacón · M. Paredes-Barquero •

S. M. Yakhine-Diop · E. Uribe-Carretero •

E. Alegre-Cortés · S. Canales-Cortés ·

M. Rodríguez-Arribas · R. A. Gonzalez-Polo $(\triangle) \cdot$

J. M. Fuentes $(\bowtie) \cdot$ M. Niso-Santano $(\bowtie)$

Instituto Universitario de Investigación Biosanitaria de

Extremadura (INUBE), Cáceres, Spain

e-mail: rosapolo@unex.es

e-mail: jfuentes@unex.es

e-mail: mnisosan@unex.es

A. Bargiela $\cdot$ M. Sabater-Arcis · R. Artero

Translational Genomics Group, Incliva Health Research Institute, Valencia, Spain

\section{A. Bargiela \\ e-mail: ariadna.bargiela@uv.es \\ M. Sabater-Arcis \\ e-mail: maria.sabater@uv.es}

\section{R. Artero}

e-mail: ruben.artero@uv.es

\section{A. Bargiela · M. Sabater-Arcis · R. Artero}

Interdisciplinary Research Structure for Biotechnology and Biomedicine (ERI BIOTECMED), University of Valencia,

Valencia, Spain showing that autophagy induction plays a critical role in the beneficial health effects of QBA.

Keywords Autophagy · Longevity ·

Neurodegeneration · Parkinson's disease - QBA · SIRT1

\section{Introduction}

Macroautophagy (hereafter referred to as autophagy) is an evolutionarily conserved degradative pathway that is rapidly upregulated to maintain cellular and organismal homeostasis when cells are exposed to stressful conditions (Mizushima and Komatsu 2011). Autophagy is characterized by the encompassing of cytoplasmic structures in a double-membrane vesicle called the autophagosome that fuses with lysosomes where their content is degraded by acidic hydrolases. This dynamic process is highly regulated by specific autophagy-related (ATG) genes (Feng et al. 2014). Moreover, various signaling pathways contribute to the outcome of autophagy
A. Bargiela $\cdot$ M. Sabater-Arcis $\cdot$ R. Artero
CIPF-INCLIVA Joint Unit, Valencia, Spain

J. Morales-García

Departamento de Biología Celular, Facultad de Medicina, Universidad Complutense de Madrid, Madrid, Spain

J. Morales-García · J. Alarcón-Gil · A. Perez-Castillo Instituto de Investigaciones Biomédicas (CSIC-UAM) "Alberto Sols" (CSIC-UAM), Madrid, Spain

J. Alarcón-Gil

e-mail: alarcon.gil.jesus@gmail.com
J. Alarcón-Gil · A. Perez-Castillo Instituto Ramón y Cajal de Investigación Sanitaria (IRYCIS), Madrid, Spain

P. J. Camello

Departamento de Fisiología, Facultad de Veterinaria, Universidad de Extremadura, Cáceres, Spain

e-mail: pcamello@unex.es

P. J. Camello

Instituto Universitario de Biomarcadores de Patologías Metabólicas, Cáceres, Spain 
regulation. Mammalian target of rapamycin (mTOR) kinase is the major signal inhibitor that prevents autophagy in the presence of growth factor and nutrient availability (Liu and Sabatini 2020). Interestingly, sirtuin 1 (SIRT1) is another nutrient sensor that can regulate autophagy through the deacetylation of ATG proteins such as ATG5, ATG6, ATG7, and ATG8/light-chain microtubule-associated protein (LC3) (Lee et al. 2008). In mammals, beclin-1 (BECN1) is a key component of class III phosphatidylinositol 3 kinase complex (PI3KIII), which plays a critical role in autophagy through the generation of phosphatidylinositol 3 phosphate (PI3P) for the nucleation of the phagophore and autophagosome biogenesis (Axe et al. 2008).

Most cells in the brain are postmitotic and require wellregulated protein quality control systems to avoid the accumulation of altered proteins and organelles (Chu 2019). In fact, a common pathological hallmark of neurodegenerative disorders is the accumulation of abnormal or misfolded proteins (Morimoto and Cuervo 2014). Increasing evidence shows that dysfunctional autophagy is associated with several neuronal disorders (Hara et al. 2006; Komatsu et al. 2006). Therefore, optimal activation of autophagy may be an essential strategy to either prevent or degrade the protein aggregation and may be promising in the treatment or prevention of neurodegeneration (Menzies et al. 2017). In subsequent years, considerable interest has emerged in the identification of autophagyregulating compounds as potential therapeutic agents in neurodegenerative diseases. In mammals, many studies have reported close relationships between nutritional factors and autophagy regulation (Castagnaro et al. 2016; Niso-Santano et al. 2015; Sheng et al. 2017). Caloric restriction (CR) is a strong physiological inducer of autophagy (Mercken et al. 2013). The signaling pathways regulating CR-mediated autophagy have been extensively studied. These pathways include the inhibition of mTOR activity and the activation of AMP-activated protein kinase (AMPK) and SIRT1 (Cohen et al. 2004; Efeyan et al. 2015; Kim et al. 2011). Furthermore, BECN1 is essential for activating the pro-autophagic PI3K-III complex in response to nutrient starvation (McKnight and Zhenyu 2013). In fact, $\mathrm{CR}$ and several natural compounds that mimic the biochemical effect of CR have been shown to promote beneficial effects and longevity (Madeo et al. 2019).

Natural bee products including honey, propolis, and royal jelly (RJ) have received increasing interest due to their therapeutic properties (Pasupuleti et al. 2017). RJ and its derived compounds possess biological activities that improve health and enhance longevity in several animal models (Honda et al. 2011; Inoue et al. 2003; Kunugi and Mohammed Ali 2019; Qiu et al. 2020; Wan et al. 2018; Xin et al. 2016). It has been reported that the lipid fraction of RJ, including 10-hydroxy-2-decenoic acid $\left(10-\mathrm{H}_{2} \mathrm{DA}\right.$, namely, queen bee acid (QBA)) and 10-hydroxydecanoic acid (10-HDA), may be responsible for its bioactive properties (Yang et al. 2010). QBA is the exclusive fatty acid of RJ, representing $40 \%$ of total fatty acid composition, and it is used as a quality marker of RJ (Li et al. 2013). Several studies have reported that QBA has anti-inflammatory, anticancer, and anti-angiogenic activities (Izuta et al. 2009; Peng et al. 2017; Sugiyama et al. 2012; Yang et al. 2018; You et al. 2019a). There are no strong evidences indicating that peripheral QBA crosses the blood-brain barrier (BBB); however, it has been shown that its administration to animal models promotes neurogenesis and neuronal health (Hattori et al. 2007). Although several studies have investigated the biological effects of QBA, its mechanism of action has not been fully elucidated. To address this issue, the present study investigated whether QBA exerts its healthy effects through autophagy induction by activating the molecular mechanisms of CR.

\section{Results}

Discovery of QBA as an autophagy modulator

To determine whether QBA induces autophagy, we exposed H4 (Fig. 1a, b) and glial U251 (Fig. S1a) cells to several concentrations of this fatty acid followed by measurement of lipidated LC3 (LC3-II) levels by Western blotting. We found that QBA treatment enhanced the amount of LC3-II compared to control cells in both cell lines. Next, we monitored autophagy flux in H4 cells that stably express a green fluorescent protein (GFP)-LC3 chimera treated with QBA in the presence or absence of Bafilomycin A1 (BAF.A1, a lysosomal inhibitor) at different time points. We observed that QBA increased LC3 puncta compared to basal conditions, which were further significantly enhanced in the presence of BAF.A1 at 2 and $4 \mathrm{~h}$ of treatment (Fig. $1 \mathrm{~d}, \mathrm{e})$. These results were confirmed in SH-SY5Y cells by Western blotting using BAF.A1 (Fig. 1c and fig. S1b). Similar to QBA, 10-hydroxydecanoic acid (HDA) also increased autophagy flux in H4-GFP-LC3 cells (Fig. S1c, d). In addition, QBA increased the 


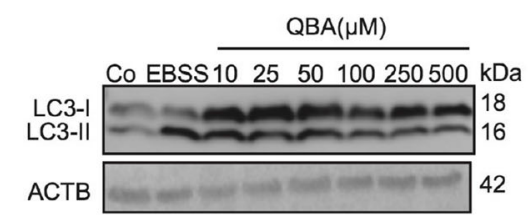

b

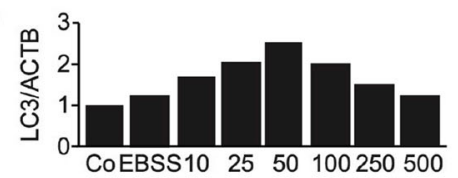

d

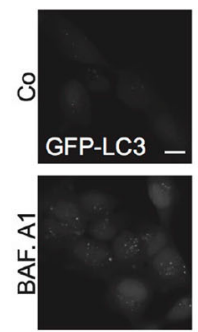

Time (h) 0
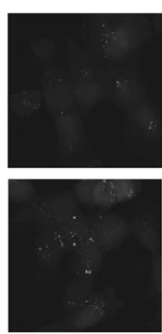

0.5

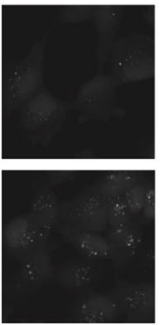

1
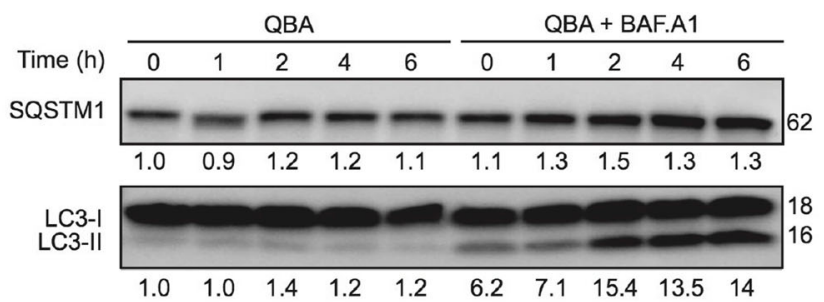

ACTB

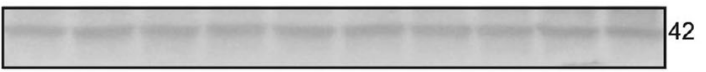

e

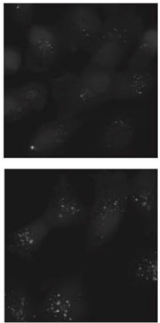

2

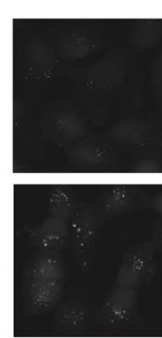

4

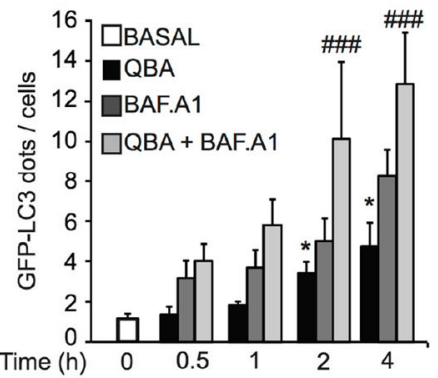

f
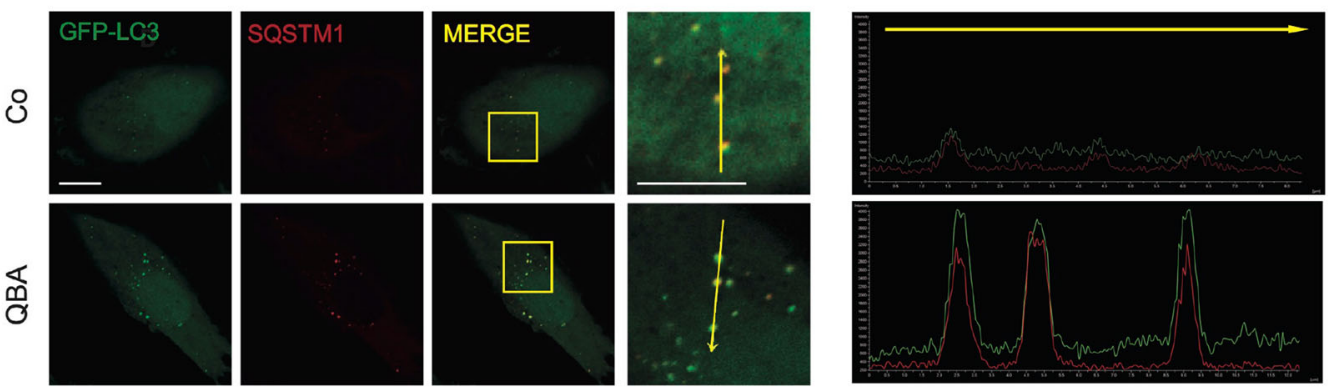

g

h

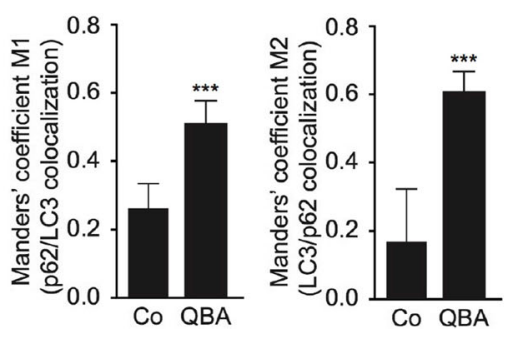

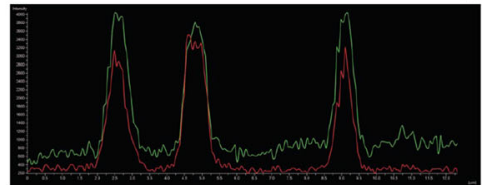

i
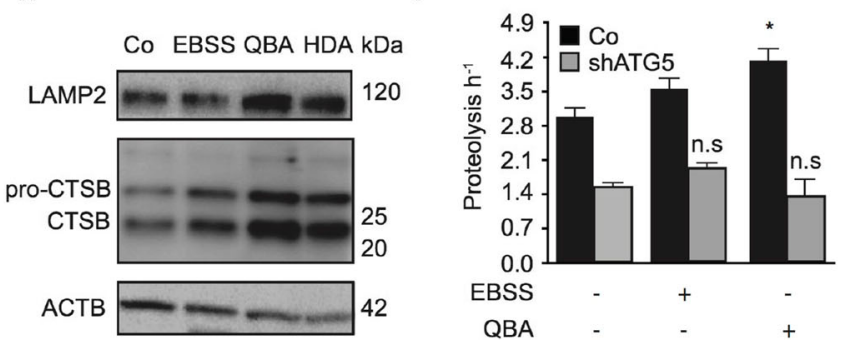

colocalization of p62 with LC3-positive vacuoles (Fig. 1f, g) in GFP-LC3 H4 cells. This autophagy activation induced by QBA was accompanied by an increase in the number of lysosomes. As shown in Fig. 1h, QBA treatment promoted the accumulation of lysosomalassociated membrane protein-2 (LAMP2) and the Cathepsin $\mathrm{B}$ mature isoform suggesting enhanced lysosomal biogenesis. To confirm the effect of QBA on 
Fig. 1 Induction of autophagy by QBA. (a-b) H4 cells were cultured in control conditions (Co), incubated with nutrient-free (EBSS) medium, or treated with QBA $(10,25,50,100,250$, and $500 \mu \mathrm{M})$ for $4 \mathrm{~h}$ followed by the assessment of LC3 lipidation by Western blot. ACTB was used as a loading control, and densitometry was employed to quantify the abundance of lipidated LC3 (LC3-II). (c) SH-SY5Y and (d) H4 cells stably expressing GFPLC3 were maintained in control conditions, treated with QBA (50 $\mu \mathrm{M})$ alone or in combination with $100 \mathrm{nM}$ Bafilomycin A1 (BAF.A1) at the indicated times. (c) LC3 lipidation and SQSTM1 degradation were assessed by immunoblotting. ACTB levels were used as a loading control. Densitometry was employed to quantify the abundance of LC3-II and SQSTM1. (e) The number of cytoplasmic GFP-LC3 ${ }^{+}$dots per cell was quantified by fluorescence microscopy in H4-GFP-LC3 cells. Scale bar $=10 \mu \mathrm{m}$. Data are the means \pm SD of at least three independent experiments $\left({ }^{*} \mathrm{p}<0.05\right.$

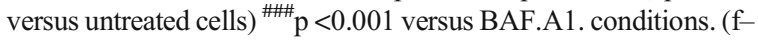
g) H4-GFP-LC3 cells were maintained in control conditions (Co) or $50 \mu \mathrm{M}$ QBA for $4 \mathrm{~h}$. Thereafter, p62-LC3 colocalization was detected by immunofluorescence. (f) Representative confocal images of GFP-LC3 (green) and p62 (red) and plots of fluorescence represent the distribution of fluorescence intensities along the line profile (yellow) Arrows indicate localized or unlocalized LC3 to p62. Scale bar $=10 \mu \mathrm{m}$. $(\mathrm{g})$ The graphs represent the average of the Mander's coefficient for the colocalization of p62 to LC3 (M1) or LC3 to p62 (M2) plus standard deviation. Symbols indicate significance at $* * * \mathrm{p}<0.001$ versus untreated cells. (h) SH-5YSY cells were cultured in control conditions (Co), EBSS medium, or treated with $50 \mu \mathrm{M}$ QBA or 10HDA for $4 \mathrm{~h}$ followed by the assessment of LAMP2 and Cathepsin B levels by immunoblotting. ACTB was used as a loading control. (i) Control (Co) and shATG5 SH-SY5Y cells were cultured in control conditions (Co), incubated with EBSS medium or treated with $50 \mu \mathrm{M}$ QBA for $4 \mathrm{~h}$. Longlived protein degradation was determined by pulse chase assay using radioactive valine as indicated in the "Materials and methods" section. Columns indicate means \pm SD. Symbols indicate significant, $* \mathrm{p}<0.05$ and non-significant (n.s.) at $\mathrm{p}>0.05$ versus untreated cells.

the induction of autophagy, the catabolism of long-lived proteins was determined by a pulse chase assay. As shown in Fig. 1i, QBA treatment remarkably enhanced the turnover of labeled long-lived proteins. Moreover, the depletion of ATG5 in SH-SY5Y cells exhibited a decreased long-lived protein degradation (Fig. 1i) and a diminution of LC3-II levels in both atg5-deficient SHSY5Y/MEF cells (Fig S1e, f). Similar results were observed in HDA-treated cells (Fig. S1e). These results clearly indicated that QBA induces autophagy in vitro in several cell lines, including neurons.
QBA-mediated autophagy is BECN1/ mTOR-dependent

To understand the molecular pathways implicated in QBA-induced autophagy, we studied the involvement of BECN1 protein and the mTOR pathway. BECN1 interacts with several ATG proteins to generate the PI3K-III complex that is required for autophagosome biogenesis (Axe et al. 2008). We studied the implications of the PI3K-III complex in the pro-autophagic activity of QBA by reducing BECN1 expression through gene silencing assays and monitoring changes in LC3 lipidation by Western blotting.

As shown in Fig. 2a and b, the depletion of BECN1 significantly reduced the lipidation of LC3 by QBA treatment in SH-SY5Y cells. Similar results were observed in HDA-treated cells. To confirm the involvement of the BECN1 complex in the activation of autophagy induced by QBA, we assessed the production of PI3P mediated by PI3K-III using U2OS cells stably expressing RFP-tagged FYVE. This domain recognizes and binds to PI3P (Yakhine-Diop et al. 2017). Figure 2c, $d$ show that QBA treatment increased RFPFYVE puncta compared to control cells. Similar to QBA, HDA treatment enhanced the number of $\mathrm{FYVE}^{+}$ dots. However, the inhibition of PI3K activity using LY294002 and 3-MA abolished the accumulation of $\mathrm{FYVE}^{+}$dots in QBA-treated cells. The same result was observed in HDA-treated cells.

mTOR negatively regulates autophagy activity in response to nutrient depletion (Liu and Sabatini 2020). Similar to EBSS, QBA treatment significantly diminished the phosphorylation levels of S6 kinase (Fig. S2a, b) and S6 protein (Fig. 2e-g) (downstream targets of mTOR), which are indicators of mTOR inhibition. In fact, the activation of mTOR kinase through the downregulation of tuberous sclerosis complex 2 (TSC2) increased the phosphorylation levels of S6 kinase and S6 protein, and diminished LC3 lipidation in QBA-treated cells, thereby inhibiting autophagic function. Taken together, these results demonstrated that QBA promotes autophagy not only by activating PI3K-III but also by suppressing mTOR activity. 
a

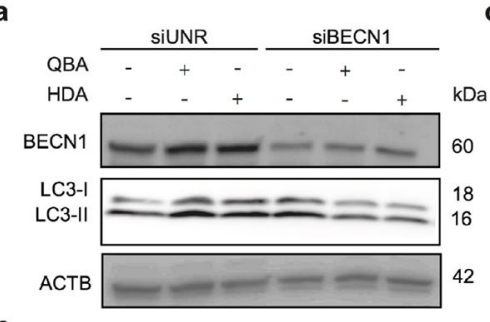

b

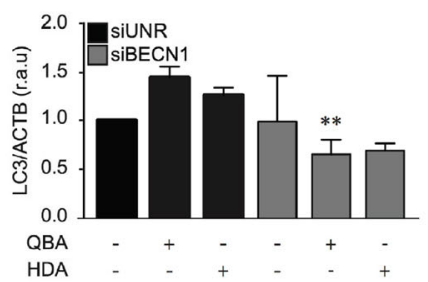

d

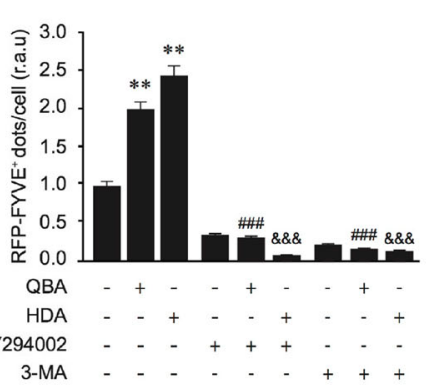

f

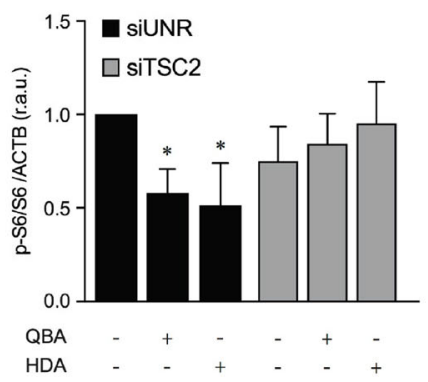

Fig. 2 Upstream regulators of autophagy induced by the lipid fraction of RJ. (a, e) SH-SY5Y cells were transfected with siUNR or with siRNA specific for BECN1 (siBECN1) or TSC2 (siTSC2) for $48 \mathrm{~h}$, and either kept in control conditions (Co) or EBSS (e) or treated with $50 \mu \mathrm{M}$ QBA or $50 \mu \mathrm{M}$ HDA for $4 \mathrm{~h}(\mathrm{a}, \mathrm{e})$. Thereafter, LC3 lipidation (a, e) and S6 phosphorylation (e) were assessed by immunoblotting. BECN1, TSC2, S6, and ACTB levels were monitored as a genotype control and to ensure equal loading of lanes, respectively. Densitometry was employed to quantify the abundance of LC3-II (b, g) and p-S6 (f). *p $<0.05$, **p $<0.01$ versus

g
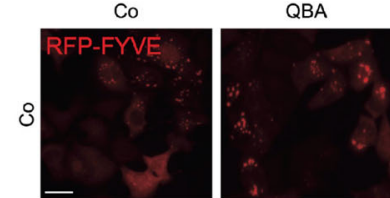

HDA
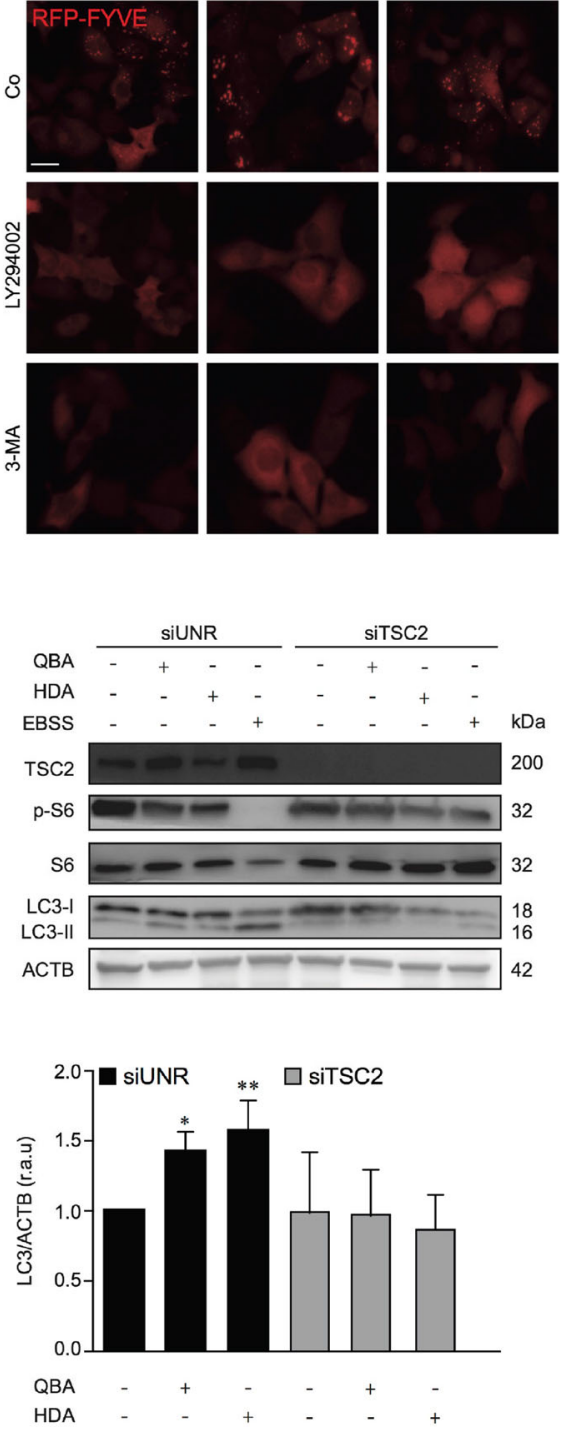

untreated cells and non-significant at $\mathrm{p}>0.05$ in siTSC2. $(\mathrm{c}, \mathrm{d})$ RFP-FYVE ${ }^{+}$expressing U2Os cells were cultured in control conditions (Co) or treated with $50 \mu \mathrm{M}$ QBA or $50 \mu \mathrm{M}$ HDA alone or in combination with $10 \mu \mathrm{M}$ LY294002 or $10 \mathrm{mM} 3$-MA. Four hours later, RFP-FYVE ${ }^{+}$dots were quantified by fluorescence microscopy. Data are normalized as the means \pm SD of at least 2

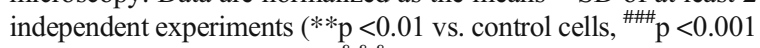
versus QBA-treated cells, ${ }^{\& \& \&} p<0.001$ versus HDA-treated cells). Scale bar $=10 \mu \mathrm{m}$. r.a.u: relative arbitrary units 


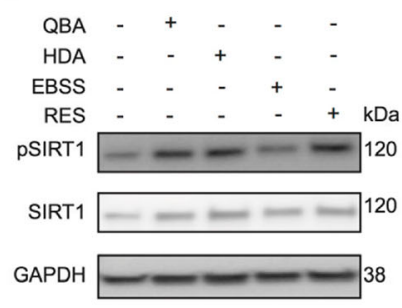

d

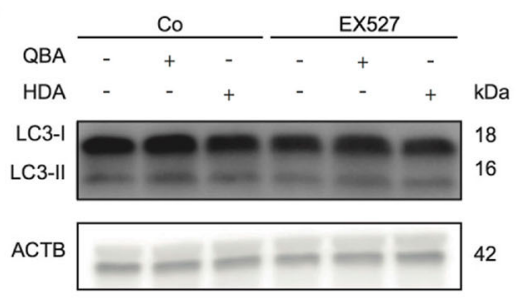

f

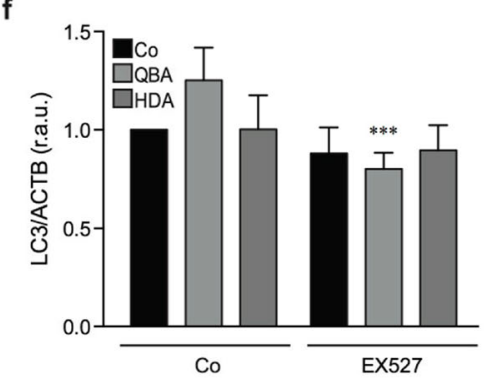

h

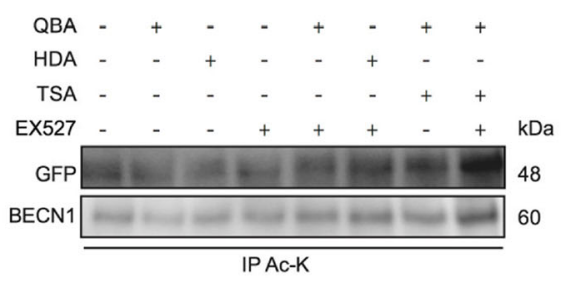

Fig. 3 Implication of SIRT1 in QBA-induced autophagy. (a, b) SH-SY5Y cells were cultured in control conditions (Co), maintained in EBSS medium, exposed to $2 \mu \mathrm{M}$ resveratrol (RES) or $50 \mu \mathrm{M}$ QBA or $50 \mu \mathrm{M} 10$-HDA (HDA) for $4 \mathrm{~h}$. Thereafter, cells were processed for the assessment of SIRT1 phosphorylation (Ser 47) (a) or SIRT1 localization detected by immunofluorescence (b). Scale bar $=10 \mu \mathrm{m}$. (c) SH-SY5Y cells were maintained in control conditions (Co), $50 \mu \mathrm{M}$ QBA, or $50 \mu \mathrm{M}$ HDA for $4 \mathrm{~h}$. Thereafter, cells were processed and the expression of SIRT1 mRNA was examined by quantitative real-time PCR. $* * p<0.01$, ***p $<0.001$ versus control cells. (d) SH-SY5Y cells were cultured in control conditions (Co) or treated with $50 \mu \mathrm{M}$ QBA or $50 \mu \mathrm{M} 10$-HDA (HDA), alone or combined with $2 \mu \mathrm{M}$ EX527 for 4 h. (e) SHSY5Y cells were transfected with a control siRNA (siUNR) or with siRNAs targeting SIRT1 (siSIRT1) for $48 \mathrm{~h}$ and either maintained in control conditions (Co) or treated with $50 \mu \mathrm{M}$ c
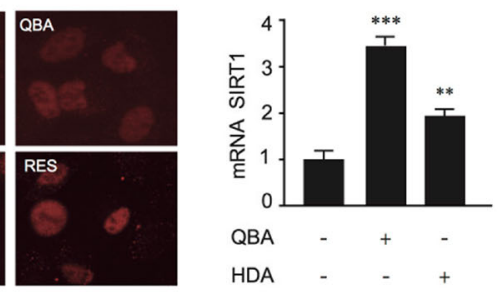

e

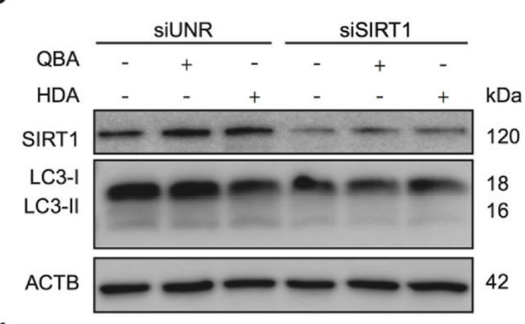

g

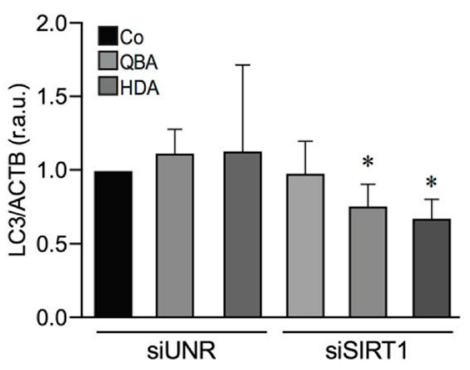

QBA or $50 \mu \mathrm{M} 10-\mathrm{HDA}$ for $4 \mathrm{~h}$. Thereafter, cells were processed for the assessment of LC3 lipidation (d, e). ACTB levels were monitored to ensure equal loading of lanes and the densitometry was employed to quantify the abundance of lipidated LC3 (LC3II) (f, g). Data are normalized as the means \pm SD of at least three independent experiments (***p $<0.001$ vs. QBA-treated cells) (f), $(* p<0.05$ versus QBA-treated siUNR cells or HDA-treated siUNR cells) (g). r.a.u: relative arbitrary units. (h) H4-GFP-LC3 cells were cultured in control conditions $(\mathrm{Co})$ or treated with $50 \mu \mathrm{M}$ QBA or $50 \mu \mathrm{M}$ HDA, alone or combined with $2 \mu \mathrm{M}$ EX527 for 4 h. $1 \mu \mathrm{M}$ TSA alone or combined with $2 \mu \mathrm{M}$ EX527 was used as a control of protein acetylation Thereafter, LC3 and BECN1 were immunoprecipitated from cell lysates with antiacetyl-lysine antibody (Ac-K) and analyzed by immunoblot using anti-GFP and anti-BECN1 antibodies. 
QBA induces SIRT1 nuclear translocation and deacetylation of ATG proteins

Previous studies show that QBA is a class I and II histone deacetylase (HDAC) inhibitor (Spannhoff et al. 2011). We analyze the role of this fatty acid in the acetylation of autophagy-related proteins through SIRT1 activation. QBA and RES enhanced the phosphorylation of SIRT1 at serine 47 and promoted its translocation to the nucleus (Fig. 3a, b). Moreover, QBA remarkably increased mRNA SIRT1 levels (Fig. 3c). To confirm the role of SIRT1 in QBA-induced autophagy, we inhibited its activity using a specific inhibitor (EX527) or by performing a gene silencing experiment (SIRT1-specific siRNA) in SHSY5Y cells. As shown in Fig. $3 d-g$, the inhibition of SIRT1 significantly reduced the levels of LC3-II compared to control cells (siUNR). Similar results were observed in cells treated with HDA. Next, we focused on determining the mechanisms by which SIRT1 promotes QBA-induced autophagy. The analysis of acetylated protein levels showed that QBA treatment enhanced the levels of acetylated lysine proteins compared to control cells and HDAtreated cells (Fig. S3a, b). Next, we performed immunoprecipitation assays with an acetylated-lysine antibody in $\mathrm{H} 4$ cells stably expressing GFP-LC3. The acetylation levels of LC3 and BECN1 were detected by Western blotting by using an anti-GFP and BECN1 antibodies, respectively. We observed that LC3 and BECN1 were deacetylated in QBA-induced autophagy, and this effect was suppressed by the inhibition of SIRT1 (Fig. 3h and fig. S3c). Similar to QBA, HDA treatment reduced the acetylation levels of LC3; however, it did not promote BECN1 deacetylation.

These findings suggested that QBA induces autophagy through deacetylation of LC3 and BECN1 proteins mediated by SIRT1 activation. Therefore, we found that QBA and HDA induced autophagy in vitro through different signaling pathways. Based on these results for these two fatty acids, we selected QBA for its capacity to induce autophagy through SIRT1-mediated deacetylation of LC3 and BECN1.

\section{QBA induces autophagy in vivo}

To analyze whether this fatty acid induces autophagy in vivo, ICR mice were injected with QBA or the saturated fatty acid HDA at a concentration of 10 $\mathrm{mg} / \mathrm{kg}$ for $4 \mathrm{~h}$. Several tissues were collected, and the analysis of LC3-II levels was monitored by immunoblotting. Figure S4a, b shows that QBA treatment remarkably induced the conversion of LC3-I to LC3-II in liver and heart tissues. Similar results were observed in HDA-treated mice or in response to nutrient deprivation. Moreover, the analysis of brain tissue showed that QBA significantly enhanced the lipidation of LC3 compare to untreated mice (Fig. 4e, f and S4c). To confirm these results, we analyzed autophagy flux in vivo after the administration of leupeptin, a protease inhibitor. LC3 only increased in liver while p62 significantly enhanced in liver and heart (Fig. 4a-d). Surprisingly, there were no changes in brain. These data indicates that QBA enhances autophagy flux in liver and heart but not in brain.

Moreover, we analyzed whether QBA triggered autophagy in Drosophila melanogaster. To this end, transgenic adult Drosophila that express GFP:Atg8a were treated with QBA and rapamycin. As shown in Fig. 4g and $\mathrm{h}$, the analysis of GFP-Atg8 by immunoblotting indicated a significant increase of the free-GFP signal in QBA-treated flies compared to control. These results were confirmed by confocal studies. Similar to rapamycin, QBA-treated flies showed an accumulation of GFP:Atg8a puncta compared to untreated flies (Fig. 4i). These results confirmed that QBA induces autophagy in mice and flies.

Inhibition of QBA-induced autophagy enhances 6-OHDA-induced toxicity

Several studies have demonstrated the pharmacological properties of QBA in animal models (Hattori et al. 2007; Honda et al. 2015; Watadani et al. 2017; Weiser et al. 2017). We analyzed whether QBA-induced autophagy exerts cytoprotective effects in a neurodegenerative model. To perform this experiment, we first examined the effect of QBA in SH-SY5Y cells treated with the neurotoxin 6hydroxy-dopamine (6-OHDA). The levels of poly (ADP-ribose) polymerase 1(PARP1), a substrate of caspase 3, was assessed by Western blotting. Our results showed that the pre-treatment of QBA notably reduced the levels of cleaved PARP1 (Fig. 5a, b) in 6-OHDA-treated cells. This decrease correlated with a reduced level of cleaved caspase 3 after QBA pretreatment in N2a cells (Fig. S5a, b). By flow cytometry, we also observed that QBA treatment decreased the cell death induced by 6-OHDA (Fig. $5 c, d$ and fig. S5c, d). These results suggest that QBA treatment partially reduces the toxicity of 6-OHDA. 
To confirm that this effect was autophagy-dependent, cells treated with 6-OHDA were exposed to QBA in the presence or not of 3-MA and we remarked that the cytoprotective effect of QBA was reduced after inhibition of autophagy in SH-SY5Y (Fig. 5c, d) and N2a cells (Fig. S5c, d). We also investigated the effect of QBA treatment in a PD mouse model. Mice were injected in the substantia nigra pars compacta $(S N p c)$ with QBA and/or 6-OHDA, and later brain tissue was prepared for immunohistochemical analysis of cell death and the inflammation response by analyzing the expression of tyrosine hydroxylase (TH) (an enzyme involved in dopamine production) as a marker of dopaminergic cells, Iba1, to label microglial cells and DAPI for nuclear staining. Triple immunofluorescence representative images (Fig. 5e and $\mathrm{f}$ ) showed that QBA injection significantly reduced the inflammatory response and cell death induced by 6-OHDA intracranial injection. These findings suggest that QBA is able to trigger autophagy and to prevent 6-OHDA-induced toxicity in vitro as well as in vivo.

QBA-induced autophagy promotes longevity in D. melanogaster

It has been shown that the induction of autophagy is required for longevity extension (Melendez et al. 2003; Nakamura and Yoshimori 2018; Pyo et al. 2013; Toth et al. 2008). Several studies have demonstrated that RJ promotes longevity in several animal models (Honda et al. 2011; Inoue et al. 2003; Xin et al. 2016). Moreover, QBA extends the lifespan in C. elegans (Honda et al. 2015). Taking this background into account and our results, we hypothesized that QBA can promote longevity through autophagy induction. To confirm this hypothesis, we performed a survival assay in Drosophila melanogaster and we observed that the flies exposed to QBA $(1.6 \mu \mathrm{M})$ showed a significant increase in lifespan compared to unexposed flies (Fig. 6a). Surprisingly, $0.16 \mu \mathrm{M}$ QBA significantly reduced lifespan in healthy female flies compared to DMSO. Next, we analyzed whether the anti-aging effect of QBA depends on autophagy using a D. melanogaster strain lacking Atg5. As shown in Fig. 6b, the increase of longevity mediated by QBA was suppressed in Atg5 ko flies compared to control flies. Indeed, QBA significantly decreased lifespan compared to the vehicle (DMSO) in both male (0.16 $\mu \mathrm{M}$ QBA) and female $(0.16 / 1.6 \mu \mathrm{M}$
QBA) ATG5 ko flies. The data suggests that, when QBA is administered to autophagy-deficient flies, the agent accelerates death. Therefore, our results showed that the QBA-mediated longevity extension is autophagydependent.

\section{Discussion}

QBA, the major lipid of RJ, has attracted increasing attention due to its beneficial effects on health. Several studies have reported its therapeutic properties; however, the precise molecular mechanisms by which QBA exerts these effects have not been elucidated (Izuta et al. 2009; Peng et al. 2017; Sugiyama et al. 2012; Yang et al. 2018; You et al. 2019a). The present study now provides compelling evidences that QBA is a novel autophagy inducer that promotes its health-promoting effects through autophagy activation.

Autophagy is an evolutionarily conserved selfdegradative mechanism responsible for the removal of aberrant misfolded proteins and damaged organelles of all eukaryotic cells. Accumulated evidence has revealed that autophagy is essential for the healthy aging of neurons (Menzies et al. 2017; Rubinsztein et al. 2012). In fact, impaired autophagy is associated with neurodegeneration (Rubinsztein et al. 2012). The discovery that the activation of autophagy by nutritional, pharmacological, or genetic interventions can reduce age-related diseases and/or extend longevity has increased scientific interest in identifying novel autophagy inducers that function as a potential therapeutic strategy to ameliorate neurodegenerative disorders. In this sense, many novel autophagy enhancers have been isolated from natural products (Eisenberg et al. 2009; Kim et al. 2013; Morselli et al. 2010; Ryu et al. 2016; Sarkar et al. 2007; Shakeri et al. 2019). RJ is a very interesting natural product because it contains health- and longevity-promoting factors that enhance the overall health, fertility, and longevity of the queen bees (Spannhoff et al. 2011; Yang et al. 2012). In addition to its beneficial effects, it has been described that RJ extends the lifespan of nematodes, flies, and mice (Honda et al. 2011; Inoue et al. 2003; Xin et al. 2016). Most studies with RJ suggest that bioactive compounds that include fatty acids and proteins might be responsible for its beneficial health effects (Fratini et al. 2016; Kunugi and Mohammed Ali 2019; Li et al. 2013; Wan et al. 2018). In this study, we have identified two fatty 
a

QBA - - - + + $++-\ldots$

Leup - - - - - - $++++++\mathrm{kDa}$ SQSTM1 62 $\mathrm{ACTB}=-\cdots=-m 42$ LC3-I m-m-m-n=m 18 LC3-II _ _ 16 АСТВ $=-\cdots-\cdots-\cdots-\cdots-\cdots$

c

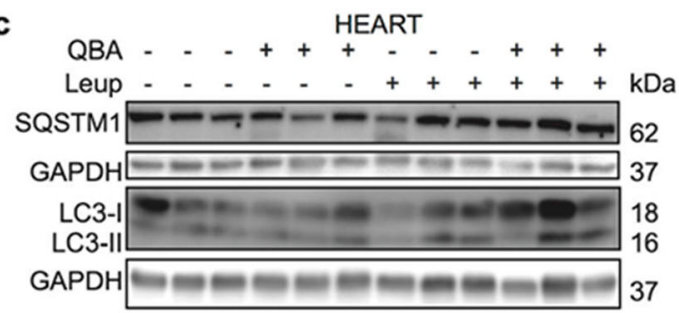

e

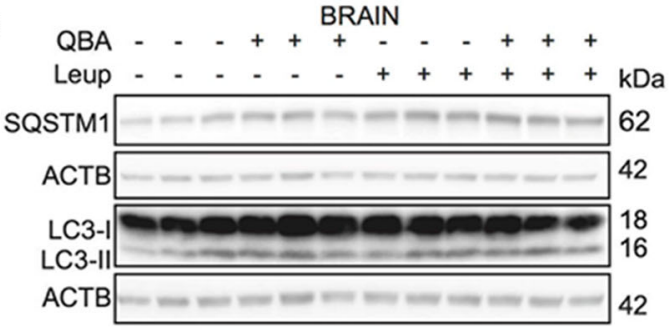

g

h
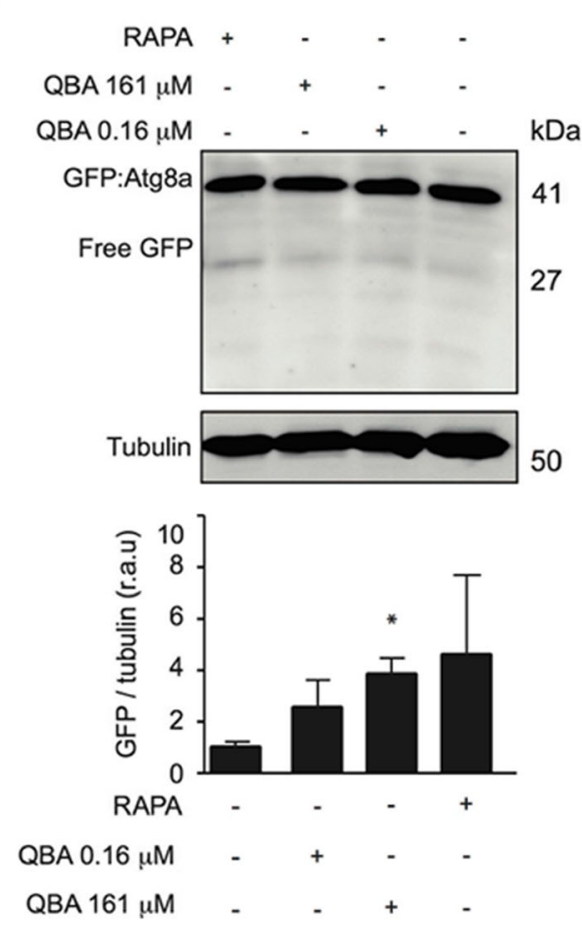

b

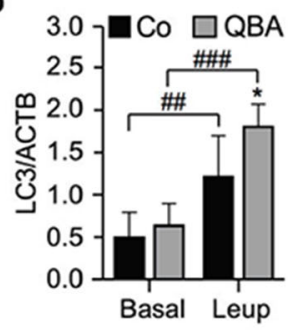

d $4.0 \square$ Co $\square \mathrm{QBA}$

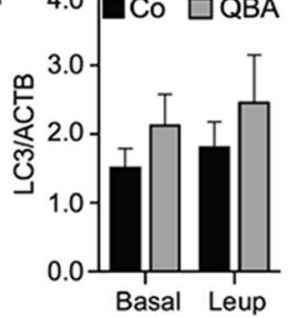

f
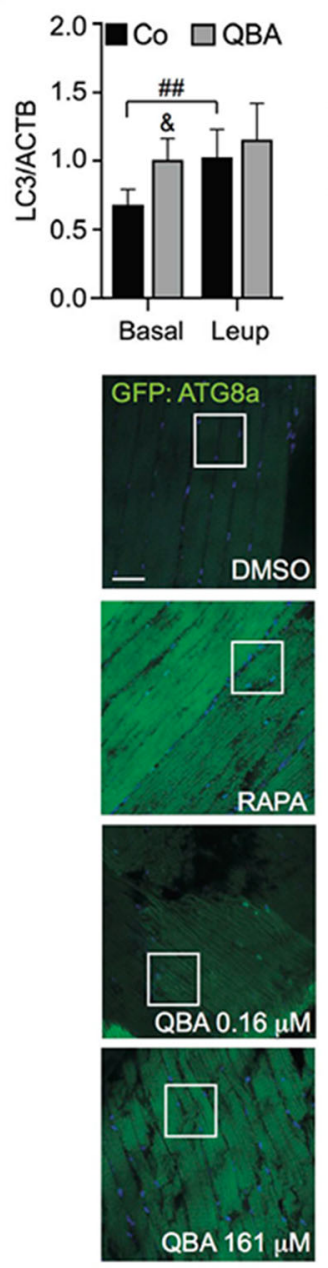
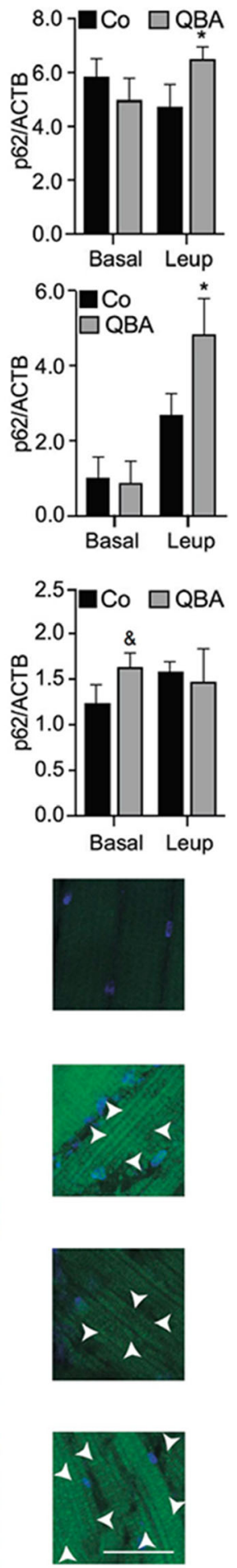
Fig. 4 Analysis of QBA-induced autophagy in vivo. (a-f) ICR mice were injected i.p. with vehicle alone or with $10 \mathrm{mg} / \mathrm{kg}$ QBA. Two hours later, mice received vehicle or $15 \mathrm{mg} / \mathrm{kg}$ leupeptin (Leup). After $2 \mathrm{~h}$, animals were euthanatized and LC3 lipidation was assessed by immunoblotting in the indicated tissues. ACTB and GAPDH levels were monitored to ensure equal loading of lanes, and densitometry was employed to quantify the abundance of lipidated LC3 (LC3-II) and p62. Data are the means \pm SD of at least 3 mice in liver and heart, and at least 5 mice in brain $\left({ }^{\#} \mathrm{p}\right.$

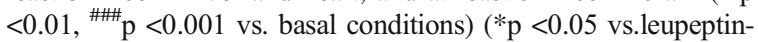
treated mice) $\left({ }^{\&} \mathrm{p}<0.05\right.$ vs. untreated mice). $(\mathrm{g}, \mathrm{h})$ Drosophila Mhc-Gal4 >UAS-GFP:Atg8a line were collected in tubes containing food supplemented, with QBA $(0.16-161 \mu \mathrm{M})$, rapamycin (RAPA, $50 \mu \mathrm{M}$ ), or DMSO as vehicle. Thereafter, GFP immunodetection was performed by Western blot. Tubulin levels were monitored to ensure equal loading of lanes ( $\mathrm{g}$ ), and densitometry was employed to quantify the abundance of GFP-Atg8a (h). Data are the means \pm SD of 3 independent experiments $(* p$ $<0.05$ vs. untreated flies). (i) Confocal fluorescence images of GFP immunostaining (green) in fly thoraces are shown. Nuclei were counterstained with DAPI (blue). Scale bar $=20 \mu \mathrm{M}$.

acids isolated from $\mathrm{RJ}$ that function as novel autophagic enhancers (QBA and HDA); however, QBA was more efficient. We found that QBA triggers autophagy in neuronal cell lines and animal models (Fig. 1 and fig. 4). The activation of autophagy mediated by QBA is BECN1 and mTOR-dependent (Fig. 2). Moreover, QBA deacetylates ATG proteins through SIRT1 activation (Fig. 3). Interestingly, QBA-mediated autophagy exerts neuroprotective effects in models of 6-OHDAmediated toxicity (Fig. 5) and extends the lifespan of D. melanogaster (Fig. 6).

Despite the numerous studies that reported the therapeutic properties promoted by QBA, the mechanism of action of this fatty acid is currently unknown. Previous studies have reported that several fatty acids trigger autophagy in multiple cell types (Chen et al. 2018; Gwon et al. 2017; Niso-Santano et al. 2015). In this study, we analyzed whether the health beneficial properties of QBA and its lifespan extension effects occur through the activation of autophagy. Remarkably, QBA enhances the recruitment of LC3 to the autophagosome membrane, increases lysosomal activity, and promotes the degradation of long-lived proteins in neuronal cell lines. One of the first events in autophagy is autophagosome formation. In fact, the PI3K-III complex regulates the size and rate of production of autophagosomes through PI3P synthesis in response to nutrient starvation (McKnight and Zhenyu 2013). Our results showed that QBA regulates the initiation step of autophagosome biogenesis through PI3P synthesis and
BECN1. Although PI3K-III is responsible for most PI3P synthesis, mammalian cells can produce PI3P through alternative mechanisms (Vicinanza et al. 2015). However, in our model, the production of PI3P depends on PI3K-III because LY294002 and 3-MA treatment (an inhibitor of PI3K-III) decreased FYVEinduced QBA.

mTOR is a major upstream signaling pathway that induces autophagy in mammalian cells (Laplante and Sabatini 2012). This protein regulates autophagy in response to nutrient availability (Gonzalez and Hall 2017). mTOR activity is regulated by the PI3K/AKT signaling pathway and AMPK activation (Gwinn et al. 2008; Sarbassov et al. 2005). Previous studies have demonstrated that QBA can alleviate neuroinflammation by the activation of the AMPK pathway and the downstream PI3K/AKT pathway (You et al. 2019a). Our results confirmed the activation of AMPK in QBA-treated cells (data not shown) and the inactivation of mTOR. Moreover, the activation of mTOR inhibits QBA-induced autophagy.

In parallel, QBA is a novel SIRT1 activator and induces autophagy through the deacetylation of ATG6 and ATG8 proteins. SIRT1 is (a NAD-dependent histone deacetylase) a class III histone deacetylase that functions as a key metabolic/energy sensor and promotes autophagic responses by the deacetylation of several ATG proteins under nutrient deprivation conditions (Cohen et al. 2004; Lee et al. 2008). It has been reported that QBA is an inhibitor of histone deacetylase (Spannhoff et al. 2011). Consistent with this, QBA treatment increased protein acetylation levels and stimulated the phosphorylation of SIRT1 (Fig. 3). Consequently, its translocation to the nucleus enhanced the deacetylation of key ATG proteins such as LC3 and BECN1. All these effects were prevented by the inhibition of SIRT1. It has been reported that autophagy is activated under nutrient stress conditions through SIRT1-Forkhead protein O 1 (FOXO1)-dependent mechanisms (Brunet et al. 2004; Nemoto et al. 2004). FOXO1 activity is regulated by its acetylation status. The acetylation of FOXO-1 can lead to inhibition of autophagic responses, while its deacetylation by SIRT1 activates autophagy (Brunet et al. 2004; Nemoto et al. 2004). A recent study demonstrated that QBA improved lipopolysaccharide-induced neuroinflammation in rodents via autophagy induction (You et al. 2019b). Given that FOXO1 is implicated in QBA-mediated autophagy, 
a

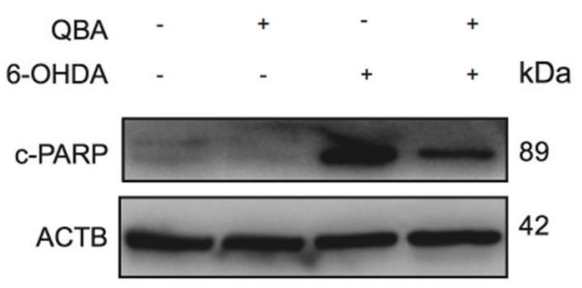

C

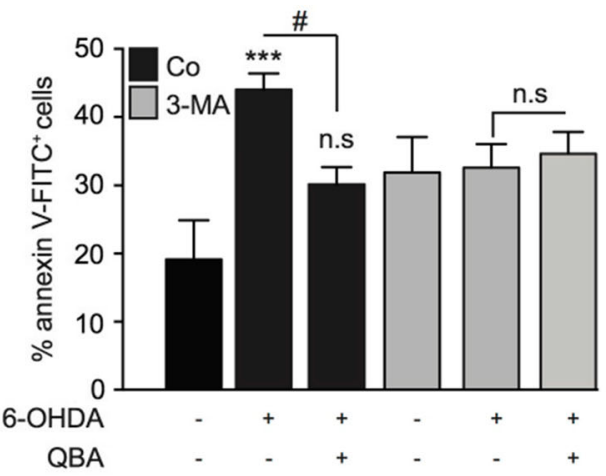

b

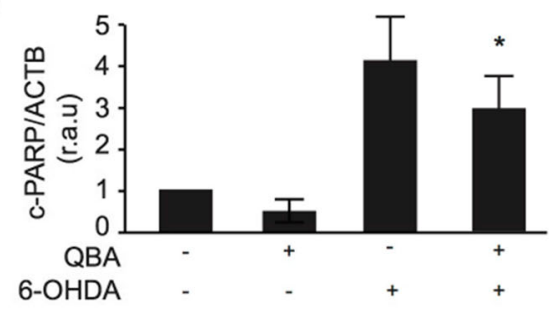

d

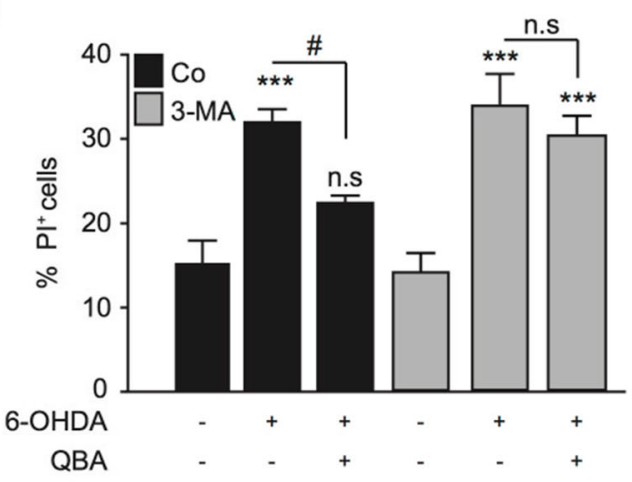

e
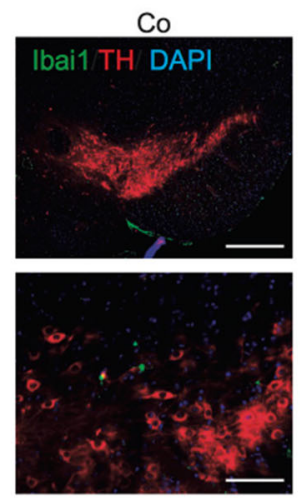

f

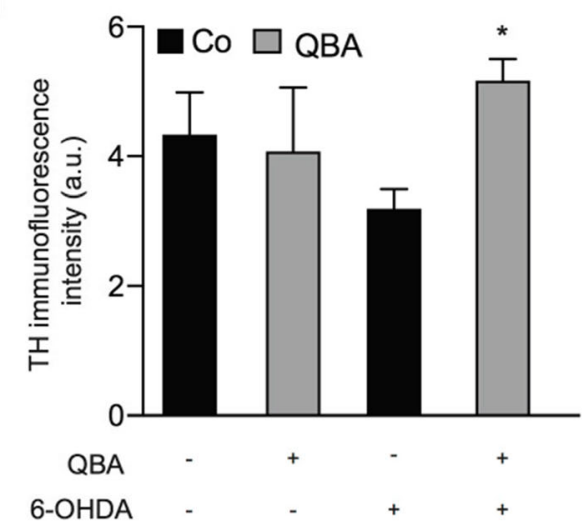

QBA
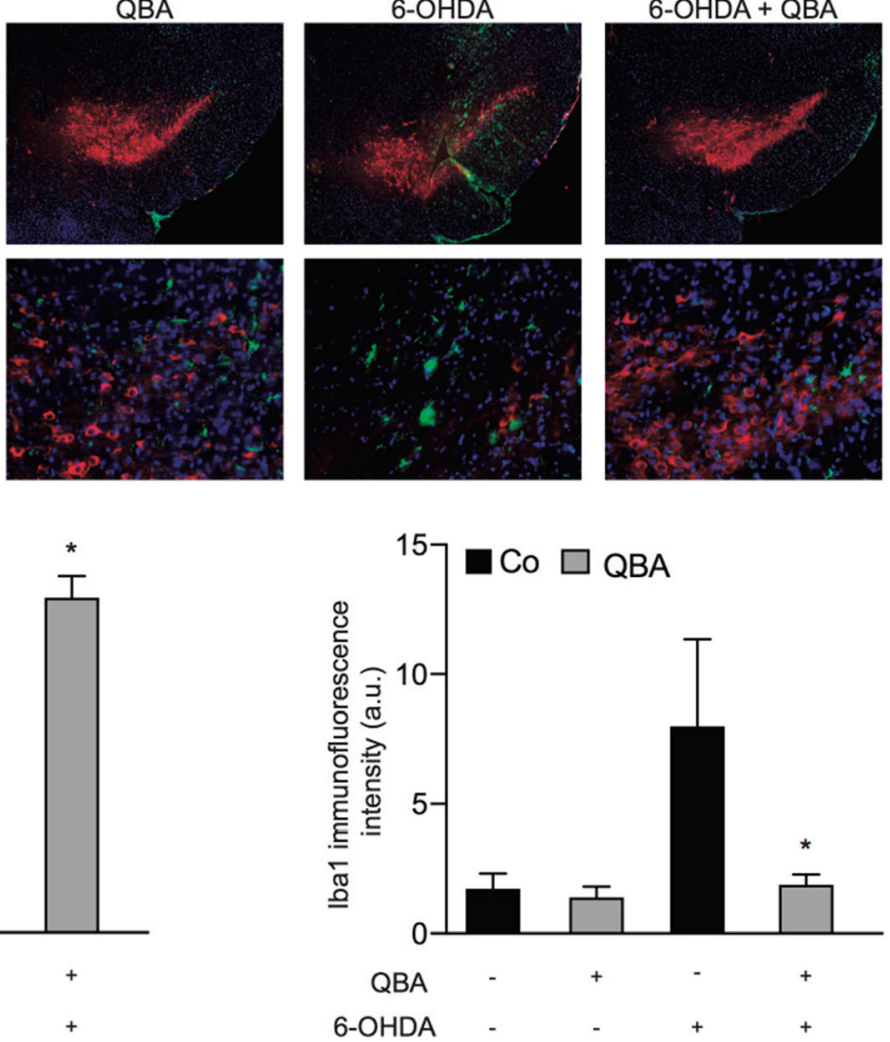
Fig. 5 QBA decreases 6-OHDA-mediated toxicity in an autophagy-dependent manner. (a-d) SH-SY5Y cells were cultured in control conditions (Co), or pre-treated with $50 \mu \mathrm{M}$ QBA for $24 \mathrm{~h}$, followed by the treatment with $35 \mu \mathrm{M}$ 6-hydroxydopamine (6-OHDA) alone $(a, b)$ or combined with $10 \mathrm{mM} 3$-methyladenine (3-MA) for $18 \mathrm{~h}$ (c, d). Cleaved PARP (c-PARP) was assessed by immunoblotting. ACTB was used as a loading control (a) and densitometry was employed to quantify the abundance of c-PARP (b). *p $<0.05$ compare with 6-OHDA-treated cells (c, d). The percentage of Annexin V-FITC-positive cells (c) and PI-positive cells (d) were evaluated by flow cytometry ( $n=10000$ events). Columns indicate means \pm SD. Symbols indicate significant, $* * * p<0.001$, and non-significant (n.s.) compared with the respective untreated groups, ${ }^{\#} \mathrm{p}<0.05$, compare with 6-OHDA-treated cells (c,d). (e) QBA treatment reduces microglial activation in the $\mathrm{SNpc}$ with 6-OHDA injections. Mice were injected with PBS (Co) or QBA alone or in combination with 6-OHDA. Animals were sacrificed, and the brains were processed by immunofluorescence. Immunostaining showing the expression of the microglial marker Ibail (green), tyrosine hydroxylase (TH, red), and DAPI (blue) in the $S N p c$ (e). Scale bar $=200 \mu \mathrm{m}$. The quantification of TH and Ibal intensity is shown in (f). Symbols indicate significant $* p$ $<0.05$ compared with 6-OHDA-treated mice.
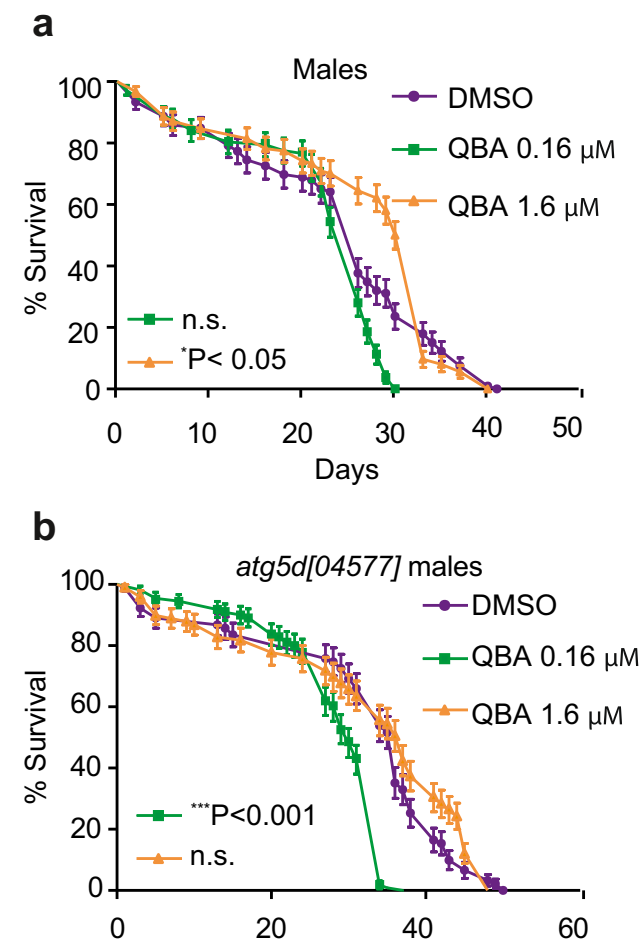

Fig. 6 QBA enhances longevity in flies. (a, b) $w^{1118}$ and Atg5d [04577] flies were maintained with standard nutritive medium supplemented with QBA $(0.16-161 \mu \mathrm{M})$ or DMSO as vehicle. Survival curves were obtained using the Kaplan-Meier method. P- we surmise that SIRT1 can modulate its antiinflammatory effect through the induction of autophagy.

$\mathrm{CR}$ is one of the most effective strategies to improve health and survival in several animal models through autophagy activation. Notably, CR regulates autophagy through nutrient-sensing signaling proteins including mTOR, AMPK, and SIRT1 (Cohen et al. 2004; Efeyan et al. 2015; Kim et al. 2011). Given that QBA activates the same signaling pathways, it could be considered as a CRM compound. In addition, previous studies with RJ or its bioactive component QBA have demonstrated its effect on longevity in several species (Honda et al. 2015; Honda et al. 2011; Inoue et al. 2003; Xin et al. 2016). In our work, we showed that QBA treatment enhances the lifespan of $D$. melanogaster, and its effect depends on autophagy activation. This result is consistent with a study showing that QBA delays aging in C. elegans in a TOR-dependent manner (Honda et al. 2015). Additionally, the administration of QBA in vivo promotes neurogenesis and neuronal health (Hattori et al. 2007). However, few studies have analyzed the effect of QBA in neurodegenerative disease. Our results showed


values were calculated with the Gehan-Breslow Wilcoxon Test. Symbols indicate significant, $* \mathrm{p}<0.05$, ***p $<0.001$ and nonsignificant (n.s.) compared with the respective untreated. 
that QBA enhances p62 in tissue samples being more significant in brain. This increase could be attributed to QBA-induced mRNA expression level (data not shown). The analysis of autophagy flux in the brain did not show statistical differences. Although leupeptin crosses the $\mathrm{BBB}$, multiple cell types exist in the brain which may show distinct autophagy responses. In fact, previous studies have already demonstrated that starvation does not provoke significant changes in brain (Mizushima et al. 2004). Moreover, the injection of QBA into $S N p c$ significantly reduced the death of dopaminergic neurons as well as the inflammatory response observed after intracranial injection of 6-OHDA. Therefore, QBA exerts protective effects against neurodegeneration in vitro and in a PD-mouse model. The inhibition of autophagy by 3MA increased the cytotoxic effect of 6-OHDA in vitro. Therefore, the autophagy-stimulating activity of QBA is associated with beneficial health effects in a PD model.

In summary, to the best of our knowledge, this study is the first to classify the two major fatty acid of Royal Jelly, QBA, and HDA, as novel autophagy inducers. Interestingly, QBA is more efficient and it acts on the same signaling pathways as CR. Most importantly, QBAinduced autophagy is able to exert a neuroprotective effect in PD models and reproduces the longevity benefits of CR in D. melanogaster, suggesting that this fatty acid may be a new potential CRM.

\section{Materials and methods}

Cell lines and culture conditions Human neuroglioma H4 cells stably expressing GFP-LC3 and human osteosarcoma U2OS cells stably expressing RFPFYVE (Zhang et al. 2007) were maintained in Dulbecco's modified Eagle's medium (DMEM) high glucose, pyruvate (Gibco, D6546-500 mL) supplemented with $10 \%$ heat-inactivated fetal bovine serum (SIGMA, F7524 $500 \mathrm{~mL}$ ), $10 \mathrm{mM}$ HEPES buffer (GIBCO 15630056), $2 \mathrm{~mL}$ of penicillin $(10 \mathrm{U} / \mathrm{ml})$ and streptomycin $(100 \mu \mathrm{g} / \mathrm{mL})$ (GIBCO, SV30010), and $500 \mu \mathrm{g} / \mathrm{mL}$ G418. Human dopaminergic neuroblastoma SH-SY5Y, stable ATG5 knockdown SHSY5Y, and mouse neuroblastoma Neuro-2a (N2a) cells were collected in RPMI medium (HyClone 1640) supplemented with $10 \%$ heat-inactivated fetal bovine serum (SIGMA, F7524-500 mL), $200 \mathrm{mM} \mathrm{L-}$ glutamine, and $0.04 \mathrm{mg} / \mathrm{mL}$ gentamicin (Gibco
15750037). Wild-type (WT) and atg5 knockout embryonic mouse fibroblasts (MEFs) were maintained in DMEM (SIGMA, D6546-500 mL) supplemented with $10 \%$ heat-inactivated BGS (HyClone, sh30541.3), $200 \mathrm{mM} \mathrm{L-glutamine,} \mathrm{and} 10 \mathrm{U} / \mathrm{mL}$ penicillin and streptomycin $(100 \mu \mathrm{g} / \mathrm{mL})$ (GIBCO, SV30010). The cells were cultured in 75 or 150 tissue culture flasks (Thermo Scientific ${ }^{\text {TM }}$ 130190) and maintained in $5 \% \quad \mathrm{CO}_{2} / 95 \%$ air at $37^{\circ} \mathrm{C}$ under saturating humidity. Cells were passaged every $24-48 \mathrm{~h}$.

Reagents and chemical treatment Cells grown to $75 \%$ confluency were seeded in 6-, 24-, or 96-well plates. After $24 \mathrm{~h}$, cells were treated with QBA (\#cay10976$500 \mathrm{mg}$, Vitro) and HDA (\#379700, Sigma-Aldrich) at different concentrations for 2-4 h. We used serum-free Earle's balanced solution salt (EBSS, \#E2888, SigmaAldrich), rapamycin (RAPA, $1 \mu \mathrm{M}$, \#R-5000, LcLabs), or resveratrol (RES, $2 \mu \mathrm{M}$, \#1418, Tocris) for $4 \mathrm{~h}$ to induce autophagy and 3-methyladenine (3-MA) for $1 \mathrm{~h}$ (10 mM, \#m9281, Sigma-Aldrich), bafilomycin A1 (BAF.A1,100 nM, \#b1080, LcLabs), or LY294002 (10 $\mu \mathrm{M}$, \#1130, Biogen) for $4 \mathrm{~h}$ to inhibit autophagy. Cells were treated with EX527 for $4 \mathrm{~h}(2 \mu \mathrm{M}$, \#E7034, Sigma-Aldrich) to inhibit the protein deacetylase SIRT. 6-Hydroxydopamine (6-OHDA, $35 \mu \mathrm{M}$, \#H116, Sigma-Aldrich) was added to the medium for $20 \mathrm{~h}$ and Trichostatin A (TSA, $1 \mu \mathrm{M}$, \#T8552, Sigma-Aldrich) was added to the medium for $4 \mathrm{~h}$ to the inhibition of class I and II HDACs.

Protein isolation and Western blotting analysis To isolate proteins, cells were collected and lysed in a buffer containing $0.5 \% \mathrm{NP}-40,100 \mathrm{mM}$ Tris $\mathrm{HCl}, 300 \mathrm{mM}$ $\mathrm{NaCl}, 1 \% \mathrm{SDS}$, and protease (\#11836170001, Roche) and phosphatase inhibitors (PhosSTOP (\#4900837001, Roche). The tissue samples were lysed with lysis buffer containing $150 \mathrm{mM} \mathrm{NaCl}, 7.5 \mathrm{mM}$ Tris $\mathrm{HCl} \mathrm{pH} 7.5$, $1 \mathrm{mM}$ EDTA and complemented with protease and phosphatase inhibitors. Total protein concentration was measured by the bicinchoninic acid assay (\#B9643-1L, Sigma-Aldrich) according to the manufacturer's instructions. Protein samples $(20 \mu \mathrm{g}$ for each samples) were subjected to an electrophoresis on $12 \%$ Tris-Glycine Mini-PROTEAN TGXTM gels (10 or 15 well, BioRad) or Criterion TGX Gels (18 well, Bio-Rad) and electrotransferred onto PVDF membranes (\#1620177, Bio-Rad). Later, membranes were blocked (1h at RT) with $10 \%(\mathrm{w} / \mathrm{v})$ fat-free milk in Tris-buffered saline 
(10 mM Tris/HCl pH 7.5, $150 \mathrm{mM} \mathrm{NaCl)} \mathrm{containing}$ 0.2\% Tween-20 (\#P5927, Sigma-Aldrich) (TBST). Membranes were washed with TBST 1X and incubated overnight at $4{ }^{\circ} \mathrm{C}$ with the corresponding primary antibodies specific to AcK (\#9441, Cell Signaling Technologies), ATG5 (\#1672630s Cell Signaling Technologies), ACTB (\#ab49900, Abcam), BECN1 (\#sc-11427, clone H-300, Santa Cruz Biotechnology Inc), Cathepsin B (\#ab58802, Abcam), cleaved caspase-3 (Asp175, \#9661, Cell Signaling Technologies), cleaved PARP (Asp214, \#9541, Cell Signaling Technologies), GAPDH (NG1740950, Millipore), GFP (\#29565, Cell Signaling Technologies), LAMP2 (sc18822, Santa Cruz Biotechnology Inc.), LC3-B (\#L7543, Sigma-Aldrich), PARP (\#9542, Cell Signaling Technologies), p62/ SQSTM1 (\#H00008878-MO1, Abnova), pS6 ribosomal protein (Ser235/236, \#4858, Cell Signaling Technologies), pSIRT1 (Ser 47, \#2314, Cell Signaling Technologies), pS6 kinase (Thr389, \#9205 Cell Signaling Technologies), S6 ribosomal protein (\#2317, Cell Signaling Technologies), S6 kinase (\#9202, Cell Signaling Technologies), SIRT1 (\#9475, Cell Signaling Technologies), and TSC2 (\#3612, Cell Signaling Technologies). After several washing steps in TBST 1X, the membranes were incubated with their respective HRP-conjugated secondary antibodies (1:10000) (\#170-6515 and \#170-5047, Bio-Rad, for rabbit and mouse antibodies, respectively) for $1 \mathrm{~h}$ at room temperature. Immunoreactive bands were visualized with ECL substrate (ThermoScientific, 32106) and chemiluminescence images were captured by an Amersham Imager 600 (GE Heathcare). Band intensities were quantified using ImageJ software (NIH), establishing ACTB, GAPDH, or tubulin protein levels as a loading control.

RNA interference Once cells reached 50\% confluence, cells were transfected with siRNAs targeting BECN1 (siBECN1, 50 UUCCGUAAGGAACAAGUCGGdTdT30), SIRT1 (siSIRT1, \#AM16708, Ambion), and TSC2 (siTSC2, \#5269468, Invitrogen-Life) using HiPerfect Transfection Reagent (\#509301704, Qiagen) according to the manufacturer's instructions. An irrelevant siRNA duplex (siUNR (Ambion, L/N 1602012)) was employed as a negative control. Forty-eight hours later, transfection efficiency was determined by Western blotting.

Expression analysis by real-time $R T-P C R$ Total RNA was extracted using an RNeasy mini kit (\#74104,
Qiagen). Genomic DNA contamination was eliminated from total RNA samples using an RNase-Free DNase kit (\#AMPD1-1KT, Sigma-Aldrich). RNA purity and quantity were checked with a NanoDrop. A total of 500 ng of total RNA was reverse transcribed into complementary DNA (cDNA) via QuantiTect reverse transcription (\#205311, Qiagen) in a $20-\mu$ L reaction according to the manufacturer's protocol. The resulting cDNA was amplified by real-time RT-PCR and SIRT mRNA expression was measured by a KAPA SYBR® Fast kit (\#KK4601, Kapa Biosystems) using the following primers from IDT ${ }^{\circledR}$ : GAPDH (5'-AGCC ACATCGCTGAGACA-3') and SIRT1(5'-TGCG GGAATCCAAAGGATAATTCAGTGTC-3'). The expression of the housekeeping gene GAPDH was used to normalize the result. The expression levels were determined by the $2\left(^{-\Delta \Delta \mathrm{Ct}}\right)$ ratio.

Automated fluorescence microscopy GFP-LC3 H4 and RFP-FYVE U2Os cells were plated on 96-well TC-treated Imaging Microplates (BD Falcon, Sparks, MD, USA) and cultured for $24 \mathrm{~h}$. After treatments, cells were washed in PBS, fixed (for $15 \mathrm{~min}$ at RT) with 4\% PFA, and stained with $2 \mu \mathrm{M}$ Hoechst 33342 (\#B2261, Sigma-Aldrich). After several washing steps with PBS (\#mb18201, Nzytech), cells were permeabilized with $0.1 \%$ Triton X100 (\#T9284, Sigma-Aldrich) for $10 \mathrm{~min}$ RT and blocked (for $1 \mathrm{~h}$ at RT) with bovine serum albumin (BSA)/PBS solution $(1 \mathrm{mg} / \mathrm{mL})$. Thereafter, cells were incubated with the specific primary antibody overnight at $4{ }^{\circ} \mathrm{C}$ followed by incubation with appropriate rabbit or mouse Alexa Fluor® 568 (Thermo Scientific, A11004) or 488 (Thermo Scientific, A11008)-conjugated secondary antibodies (for $1 \mathrm{~h}$ at RT). Image acquisition was done with a Cellsens IX83 molecular inverted microscope. The presence of cytoplasmic GFP-LC3 ${ }^{+}$and RFP-FYVE ${ }^{+}$dots was assessed using Ifdotmeter ${ }^{\circledR}$ software (Rodriguez-Arribas et al. 2016).

Confocal microscopic analysis GFP-LC3 H4 cells were seeded in 96-well TC-treated Imaging Microplates (BD Falcon, Sparks, MD, USA) $24 \mathrm{~h}$ before stimulation. After experimental treatments, cells were fixed with $4 \%$ PFA supplemented with DAPI (ThermoFisher, P36966). Fluorescence images were visualized using an A1 confocal imaging system mounted on an inverted Eclipse Ti microscope (Nikon Corp., Tokyo, Japan A1). LC3 and lysotracker or SQSTM1 cytoplasmic colocalization data 
were analyzed with the JACoP plugin of ImageJ software with preprogrammed macros. At least 100 hundred cells were analyzed in every experiment.

Immunoprecipitation assay For acetylation analysis, whole-cell extracts were lysed with cell lysis reagent (CelLytic $^{\text {TM }}$ M, c2978, Sigma-Aldrich) supplemented with $10 \%$ deacetylase inhibitor cocktail (\#sc362323, Santa Cruz Biotechnology Inc., Santa Cruz, CA, USA) and $10 \mu \mathrm{M}$ anacardic acid (\#A7236, Sigma-Aldrich). Two hundred micrograms of protein was incubated with Dynabeads protein G (\#1007D, Novex) for $90 \mathrm{~min}$. Once the beads attached to the nonspecificities were discarded, samples were incubated with Ac-K antibody overnight at $4{ }^{\circ} \mathrm{C}$ in agitation. Proteins were then reincubated with Dynabeads for $150 \mathrm{~min}$. Immunoprecipitated proteins were washed three times with lysis buffer and eluted with Laemmli $2 \mathrm{x}$ and SDS loading buffer $5 \mathrm{x}$ for $1 \mathrm{~min}$ at $95^{\circ} \mathrm{C}$.

Mouse experiments ICR mice were kept under controlled conditions with food and water ad libitum. Four-week-old male animals were injected intraperitoneally with either $10 \mathrm{mg} / \mathrm{kg}$ QBA or $10 \mathrm{mg} / \mathrm{kg}$ HDA or an equivalent volume of vehicle. Four hours later, mice were euthanized. To induce autophagy, animals were starved overnight. To analyze autophagic flux, mice received an intraperitoneal injection of QBA, and $2 \mathrm{~h}$ later, mice were again injected intraperitoneally with 15 $\mathrm{mg} / \mathrm{kg}$ leupeptin (in $150 \mathrm{mM} \mathrm{NaCl}$ ) or an equivalent volume of vehicle and were sacrificed $2 \mathrm{~h}$ later. Thereafter, organs were collected and processed for immunoblotting detection of LC3 lipidation. All animal experiments were approved by the Committee for Animal Experimentation of the Universidad de Extremadura and were performed in accordance with its guidelines.

In 6-OHDA studies, C57BL/6 male mice, 2 to 3 months of age, were maintained on a 12-h light/dark cycle with access to food and water ad libitum. The animal protocols used in this study were performed in accordance with institutional guidelines (Institutional Animal Care and Use Committee guidelines) and approved by the "Ethics committee for Animal Experimentation" of the Instituto de Investigaciones Biomédicas (CSIC-UAM). Animals (four mice per experimental group) were anesthetized with a mixture of ketamine $(60 \mathrm{mg} / \mathrm{kg}) /$ medetomidine $(5 \mu \mathrm{g} / \mathrm{kg})$ and placed in a stereotaxic apparatus (Koft Instruments, $\mathrm{CA})$. Mice were then injected with either $1 \mu \mathrm{g}$ of 6-OHDA dissolved in $2.5 \mu \mathrm{L}$ of PBS or QBA $(11 \mu \mathrm{g}$ in $2.5 \mu \mathrm{L}$ of
PBS) or the same dose of 6-OHDA in combination with QBA (11 $\mu \mathrm{g}$ in $2.5 \mu \mathrm{L}$ of PBS) into the right side of the $\mathrm{SNpc}$ at the following coordinates: posterior $-3.2 \mathrm{~mm}$, lateral $+2.0 \mathrm{~mm}$, and ventral $+4.7 \mathrm{~mm}$ relative to Bregma and according to the atlas of Paxinos and Watson (Paxinos 1998). Parallel injections of $2.5 \mu \mathrm{L}$ of PBS served as controls for the 6-OHDA-treated animals. To analyze the effect of autophagy inhibition, mice were anesthetized and injected with 6-OHDA (1 mg in $2.5 \mathrm{~mL}$ of PBS) or 6OHDA in combination with QBA $(11 \mu \mathrm{g}$ in $2.5 \mu \mathrm{L}$ of PBS) as described previously. After different treatments, mice were housed individually to recover and sacrificed 7 days after lesioning.

Histology and immunohistochemistry Mice were anesthetized and transcardially perfused with ice-cold PBS and 4\% paraformaldehyde (PFA). Brains were harvested, postfixed in $4 \%$ PFA at $4^{\circ} \mathrm{C}$ overnight, cryoprotected in $30 \%$ sucrose, and stored at $-80^{\circ} \mathrm{C}$. Brain tissue was cut into 30- $\mu \mathrm{m}$-thick sections using a cryostat (Cryocut 1900, Leica) and stored in a solution containing $30 \%$ ethylene glycol, $30 \%$ glycerol, and $40 \%$ phosphate buffer $0.1 \mathrm{M}$ at $-80^{\circ} \mathrm{C}$ until use. For immunohistochemistry, sections were permeabilized and blocked with $0.1 \%$ Triton X-100 and $3 \%$ goat serum in PBS for $1 \mathrm{~h}$ at room temperature followed by overnight incubation with primary antibodies against monoclonal mouse tyrosine hydroxylase $(\mathrm{TH})$ (T2928, Sigma-Aldrich) and polyclonal rabbit Ibal (01127991, Wako). After several washing steps, all sections were incubated for $1 \mathrm{~h}$ at RT with a secondary Alexa-Fluor 488 goat anti-rabbit or Alexa-Fluor 647 goat anti-mouse antibodies (1:500, Jakcson ImmunoResearch) and washed, and nuclei were stained with DAPI for $15 \mathrm{~min}$ at room temperature. Finally, coverslips were mounted in Vectashield H-1000 (Vector Laboratories). Images were acquired under a fluorescence microscope (Nikon 90i).

D. melanogaster studies $w^{1118}$, Mhc-Gal4 >UASGFP:Atg8a, Atg5d[04577] lines were obtained from the Bloomington Drosophila Stock Center (Indiana University, Bloomington, IN, USA). Mhc-Gal4 flies were described previously (Marek et al. 2000). Flies were maintained at $25{ }^{\circ} \mathrm{C}$ with standard fly food. For oral administration of QBA, a maximum of 25 1-day-old adult flies was collected in tubes containing standard food supplemented with QBA $(0.16-161 \mu \mathrm{M})$. Flies were transferred to tubes containing fresh food every 2-3 days. GFP (11814460001, Sigma-Aldrich) and $\alpha-$ tubulin (clone 12G10, Developmental Studies 
Hybridoma Bank) immunodetection in protein extracts and GFP:Atg8 in fly thoraces was performed as previously described (Bargiela et al. 2015).

D. melanogaster lifespan analyses A total of 90-100 newly emerged flies were collected in freshly prepared tubes containing standard nutritive medium with QBA (concentrations ranging from 0.16 to $161 \mu \mathrm{M}$ ), rapamycin $(50 \mu \mathrm{M})$, or DMSO as vehicle. Males and females were kept in different tubes at $29^{\circ} \mathrm{C}$. The number of dead flies was scored daily. Flies were transferred to new tubes twice a week. Survival curves were obtained using the Kaplan-Meier method and were statistically compared according to the Gehan-Breslow-Wilcoxon test $(\alpha=0.05)$.

Flow cytometry assay Cells were seeded in 24-well plates and cultured for $24 \mathrm{~h}$. After experimental interventions, cells were collected in FACS tubes (51031, Enzo laboratories) and stained with Annexin V-FITC (Immunostep, ANXVF-200T) for $15 \mathrm{~min}$ at $37^{\circ} \mathrm{C}$ and after propidium iodide $(0.1 \mathrm{mg} / \mathrm{mL}$ ) (PI, Sigma-Aldrich, P4170) was added to each tube to detect either the percentage of PI- or Annexin-positive cells using a Beckman Coulter FC500-MPL.

Degradation of long-lived proteins Cells were seeded in 6-well plates and cultured for $24 \mathrm{~h}$. After treatments, cells were maintained with $0.2 \mathrm{lCi} / \mathrm{mL}$ [14C]valine (NEC291EU050UC, PerkinElmer) for $18 \mathrm{~h}$ at $37^{\circ} \mathrm{C}$. After several washing steps in PBS (pH 7.4), the medium with the isotope-labeled amino acid was changed for a cell culture medium with L-valine (V0153, Sigma-Aldrich), first for $1 \mathrm{~h}$ (prechase), and then in combination with several experimental treatments (chase) for $4 \mathrm{~h}$. Thereafter, proteins were precipitated with $10 \%(\mathrm{v} / \mathrm{v})$ trichloroacetic acid (TCA) (T9159, Sigma-Aldrich), separated from the soluble radioactive fraction by centrifugation at $6000 \mathrm{~g}$ for $20 \mathrm{~min}$ and then dissolved in $0.2 \mathrm{~N}$ $\mathrm{NaOH}$. Finally, the long-lived protein degradation was measured by adding a small quantity of each sample into a scintillation liquid and measuring the radioactive activity with a liquid scintillation counter.

Statistical analyses Data are expressed as the mean \pm SD of triplicate determinations. Experiments were repeated at least three times, yielding similar results.
Unless otherwise indicated, comparisons among groups used unpaired Student's test, one-way ANOVA, or twoway ANOVA with the Sidak or Tukey posttest.

Supplementary Information The online version contains supplementary material available at https://doi.org/10.1007/s10565021-09625-w.

Acknowledgements We thank Prof. Guido Kroemer (Cordeliers Research Center) for the kind gift of $\mathrm{H} 4$ cells stably expressing GFPLC3 and WT and ATG5 knockout MEFs, Prof. Ana Perez-Castillo (CSIC-UAM) for stable ATG5 knockdown SH-SY5Y cells, and Prof. Junying Yuan (Harvard Medical School) for the FYVE RFPencoding construct. The authors also thank M.P. Delgado-Luceño and FUNDESALUD for helpful assistance.

Code availability Not applicable

Author contribution G. M-C and M. P-B performed and interpreted most of the experiments and helped writing the manuscript. A. B, and M. S-A performed experiments and interpreted the results. S.M.S Y-D, E. U-C, J. M-G, J. A-G, E A-C, S. C-C, P.J. C, and R.A. G-P performed experimental work. J.M. B-SP and M. R-A analyzed the data. A. P-C and R. A. designed experiments and commented on the manuscript. R.A G-P, J.M. F, and M. N-S. supervised project planning and development and commented on the manuscript. J.M. F and M N-S conceived and designed the research. M N-S analyzed the data and wrote the manuscript. All authors read and approved the final manuscript.

Funding Open Access funding provided thanks to the CRUECSIC agreement with Springer Nature. This research was supported by a grant (IB18048) from Junta de Extremadura, Spain, and a grant (RTI2018-099259-A-I00) from Ministerio de Ciencia e Innovación, Spain. This work was also partially supported by "Fondo Europeo de Desarrollo Regional" (FEDER) from the European Union. Part of the equipment employed in this work has been funded by Generalitat Valeciana and co-financed with ERDF funds (OP EDRF of Comunitat Valenciana 2014-2020). G.M-C is supported by University of Extremadura (ONCE Foundation). M.P-B is a recipient of a fellowship from the "Plan Propio de Iniciación a la Investigación, Desarrollo Tecnológico e Innovación (University of Extremadura)." S.M.S.Y-D is supported by CIBERNED. E.U-C was supported by an FPU predoctoral fellowship FPU16/00684 from Ministerio de Educación, Cultura y Deporte. A.B. was supported by a postdoctoral fellowship (APOSTD2017/077). M.S.A. was supported by a predoctoral fellowship (ACIF/2018/071) both from the Conselleria d'Educació, Investigació, Cultura i Esport (Generalitat Valenciana). E.A-C was supported by a grant (IB18048) from Junta de Extremadura, Spain. S.C-C was supported by an FPU predoctoral fellowship FPU19/04435 from Ministerio de Educación, Cultura y Deporte. J.M.B-S. P was funded by the "Ramón y Cajal" program (RYC2018-025099). J.M.F. received research support from the Instituto de Salud Carlos III, CIBERNED (CB06/05/004). M.N-S was funded by the "Ramon y Cajal" Program (RYC-2016-20883) Spain. 
Data availability The datasets generated during and/or analyzed during the current study are available from the corresponding author on reasonable request.Supplementary Information The online version contains supplementary material available at https://doi.org/10.1007/s10565-021-09625-w.

\section{Declarations}

\section{Consent to participate Not applicable}

\section{Consent for publication Not applicable}

Conflict of interest The authors declare no competing interests.

\begin{abstract}
Abbreviations $A M P K$, AMP-activated protein kinase; $A T G$, Autophagy-related; $B A F . A 1$, Bafilomycin A1; BECN1, Beclin-1; $C R$, Caloric restriction; FOXO1, Forkhead box protein $\mathrm{O}$ 1; HDA, 10-Hydroxydecanoic acid; 6-OHDA, 6Hydroxydopamine; LAMP2, Lysosomal-associated membrane protein-2; $m T O R$, Mammalian target of rapamycin; $P D$, Parkinson's disease; $P I$, Propidium iodide; $Q B A$, Queen bee acid; PI3K-III, Class III phosphatidylinositol- 3-kinase; $P I 3 P$, Phosphatidylinositol 3 phosphate; RJ, Royal Jelly; SIRT1, Sirtuin 1 or silent information regulator 2 homolog 1; S6K, S6 kinase; SQSTM1, Sequestosome 1; TSC2, Tuberous sclerosis complex 2
\end{abstract}

Open Access This article is licensed under a Creative Commons Attribution 4.0 International License, which permits use, sharing, adaptation, distribution and reproduction in any medium or format, as long as you give appropriate credit to the original author(s) and the source, provide a link to the Creative Commons licence, and indicate if changes were made. The images or other third party material in this article are included in the article's Creative Commons licence, unless indicated otherwise in a credit line to the material. If material is not included in the article's Creative Commons licence and your intended use is not permitted by statutory regulation or exceeds the permitted use, you will need to obtain permission directly from the copyright holder. To view a copy of this licence, visit http://creativecommons.org/licenses/by/4.0/.

\section{References}

Axe EL, Walker SA, Manifava M, Chandra P, Roderick HL, Habermann A, et al. Autophagosome formation from membrane compartments enriched in phosphatidylinositol 3phosphate and dynamically connected to the endoplasmic reticulum. J Cell Biol. 2008;182:685-701.

Bargiela A, Cerro-Herreros E, Fernandez-Costa JM, Vilchez JJ, Llamusi B, Artero R. Increased autophagy and apoptosis contribute to muscle atrophy in a myotonic dystrophy type 1 Drosophila model. Dis Model Mech. 2015;8:679-90.
Brunet A, Sweeney LB, Sturgill JF, Chua KF, Greer PL, Lin Y, et al. Stress-dependent regulation of FOXO transcription factors by the SIRT1 deacetylase. Science. 2004;303:2011-5.

Castagnaro S, Pellegrini C, Pellegrini M, Chrisam M, Sabatelli P, Toni S, et al. Autophagy activation in COL6 myopathic patients by a low-protein-diet pilot trial. Autophagy. 2016;12:2484-95.

Chen X, Pan Z, Fang Z, Lin W, Wu S, Yang F, et al. Omega-3 polyunsaturated fatty acid attenuates traumatic brain injuryinduced neuronal apoptosis by inducing autophagy through the upregulation of SIRT1-mediated deacetylation of Beclin1. J Neuroinflammation. 2018;15:310.

Chu CT. Mechanisms of selective autophagy and mitophagy: implications for neurodegenerative diseases. Neurobiol Dis. 2019;122:23-34.

Cohen HY, Miller C, Bitterman KJ, Wall NR, Hekking B, Kessler $\mathrm{B}$, et al. Calorie restriction promotes mammalian cell survival by inducing the SIRT1 deacetylase. Science. 2004;305:390-2.

Efeyan A, Comb WC, Sabatini DM. Nutrient-sensing mechanisms and pathways. Nature. 2015;517:302-10.

Eisenberg T, Knauer H, Schauer A, Buttner S, Ruckenstuhl C, Carmona-Gutierrez D, et al. Induction of autophagy by spermidine promotes longevity. Nat Cell Biol. 2009;11: 1305-14.

Feng Y, He D, Yao Z, Klionsky DJ. The machinery of macroautophagy. Cell Res. 2014;24:24-41.

Fratini F, Cilia G, Mancini S, Felicioli A. Royal Jelly: an ancient remedy with remarkable antibacterial properties. Microbiol Res. 2016;192:130-41.

Gonzalez A, Hall MN. Nutrient sensing and TOR signaling in yeast and mammals. EMBO J. 2017;36:397-408.

Gwinn DM, Shackelford DB, Egan DF, Mihaylova MM, Mery A, Vasquez DS, et al. AMPK phosphorylation of raptor mediates a metabolic checkpoint. Mol Cell. 2008;30:214-26.

Gwon DH, Hwang TW, Ro JY, Kang YJ, Jeong JY, Kim DK, et al. High endogenous accumulation of omega-3 polyunsaturated fatty acids protect against ischemia-reperfusion renal injury through AMPK-mediated autophagy in Fat-1 mice. Int J Mol Sci. 2017;18:2081.

Hara T, Nakamura K, Matsui M, Yamamoto A, Nakahara Y, Suzuki-Migishima R, et al. Suppression of basal autophagy in neural cells causes neurodegenerative disease in mice. Nature. 2006;441:885-9.

Hattori N, Nomoto H, Fukumitsu H, Mishima S, Furukawa S. Royal jelly and its unique fatty acid, 10-hydroxy-trans-2decenoic acid, promote neurogenesis by neural stem/ progenitor cells in vitro. Biomed Res. 2007;28:261-6.

Honda Y, Fujita Y, Maruyama H, Araki Y, Ichihara K, Sato A, et al. Lifespan-extending effects of royal jelly and its related substances on the nematode Caenorhabditis elegans. PLoS One. 2011;6:e23527.

Honda Y, Araki Y, Hata T, Ichihara K, Ito M, Tanaka M, et al. 10Hydroxy-2-decenoic acid, the major lipid component of Royal Jelly, extends the lifespan of Caenorhabditis elegans through dietary restriction and target of rapamycin signaling. J Ag Res. 2015;2015:425261.

Inoue S, Koya-Miyata S, Ushio S, Iwaki K, Ikeda M, Kurimoto M. Royal Jelly prolongs the life span of $\mathrm{C} 3 \mathrm{H} / \mathrm{HeJ}$ mice: correlation with reduced DNA damage. Exp Gerontol. 2003;38: 965-9. 
Izuta H, Chikaraishi Y, Shimazawa M, Mishima S, Hara H. 10Hydroxy-2-decenoic acid, a major fatty acid from royal jelly, inhibits VEGF-induced angiogenesis in human umbilical vein endothelial cells. Evid Based Complement Alternat Med. 2009;6:489-94.

Kim J, Kundu M, Viollet B, Guan KL. AMPK and mTOR regulate autophagy through direct phosphorylation of Ulk1. Nat Cell Biol. 2011;13:132-41.

Kim HS, Montana V, Jang HJ, Parpura V, Kim JA. Epigallocatechin gallate (EGCG) stimulates autophagy in vascular endothelial cells: a potential role for reducing lipid accumulation. J Biol Chem. 2013;288:22693-705.

Komatsu M, Waguri S, Chiba T, Murata S, Iwata J, Tanida I, et al. Loss of autophagy in the central nervous system causes neurodegeneration in mice. Nature. 2006;441:880-4.

Kunugi H, Mohammed Ali A. Royal Jelly and its components promote healthy aging and longevity: from animal models to humans. Int J Mol Sci. 2019;20:4662.

Laplante M, Sabatini DM. mTOR signaling in growth control and disease. Cell. 2012;149:274-93.

Lee IH, Cao L, Mostoslavsky R, Lombard DB, Liu J, Bruns NE, et al. A role for the NAD-dependent deacetylase Sirt1 in the regulation of autophagy. Proc Natl Acad Sci U S A. 2008;105:3374-9.

Li X, Huang C, Xue Y. Contribution of lipids in honeybee (Apis mellifera) royal jelly to health. J Med Food. 2013;16:96-102.

Liu GY, Sabatini DM. mTOR at the nexus of nutrition, growth, ageing and disease. Nat Rev Mol Cell Biol. 2020;21:183-203.

Madeo F, Carmona-Gutierrez D, Hofer SJ, Kroemer G. Caloric restriction mimetics against age-associated disease: targets, mechanisms, and therapeutic potential. Cell Metab. 2019;29: 592-610.

Marek KW, Ng N, Fetter R, Smolik S, Goodman CS, Davis GW. A genetic analysis of synaptic development: pre- and postsynaptic dCBP control transmitter release at the Drosophila NMJ. Neuron. 2000;25:537-47.

McKnight NC, Zhenyu Y. Beclin 1, an essential component and master regulator of PI3K-III in health and disease. Curr Pathobiol Rep. 2013;1:231-8.

Melendez A, Talloczy Z, Seaman M, Eskelinen EL, Hall DH, Levine B. Autophagy genes are essential for dauer development and life-span extension in C. elegans. Science. 2003;301:1387-91.

Menzies FM, Fleming A, Caricasole A, Bento CF, Andrews SP, Ashkenazi A, et al. Autophagy and neurodegeneration: pathogenic mechanisms and therapeutic opportunities. Neuron. 2017;93:1015-34.

Mercken EM, Crosby SD, Lamming DW, JeBailey L, KrzysikWalker S, Villareal DT, et al. Calorie restriction in humans inhibits the PI3K/AKT pathway and induces a younger transcription profile. Aging Cell. 2013;12:645-51.

Mizushima N, Komatsu M. Autophagy: renovation of cells and tissues. Cell. 2011;147:728-41.

Mizushima N, Yamamoto A, Matsui M, Yoshimori T, Ohsumi Y. In vivo analysis of autophagy in response to nutrient starvation using transgenic mice expressing a fluorescent autophagosome marker. Mol Biol Cell. 2004;15:1101-11.

Morimoto RI, Cuervo AM. Proteostasis and the aging proteome in health and disease. J Gerontol A Biol Sci Med Sci. 2014;69(Suppl 1):S33-8.
Morselli E, Maiuri MC, Markaki M, Megalou E, Pasparaki A, Palikaras K, et al. Caloric restriction and resveratrol promote longevity through the Sirtuin-1-dependent induction of autophagy. Cell Death Dis. 2010;1:e10.

Nakamura S, Yoshimori T. Autophagy and longevity. Mol Cell. 2018;41:65-72.

Nemoto S, Fergusson MM, Finkel T. Nutrient availability regulates SIRT1 through a forkhead-dependent pathway. Science. 2004;306:2105-8.

Niso-Santano M, Malik SA, Pietrocola F, Bravo-San Pedro JM, Marino G, Cianfanelli V, et al. Unsaturated fatty acids induce non-canonical autophagy. EMBO J. 2015;34:1025-41.

Pasupuleti VR, Sammugam L, Ramesh N, Gan SH. Honey, propolis, and royal jelly: a comprehensive review of their biological actions and health benefits. Oxidative Med Cell Longev. 2017;2017:1259510.

Paxinos GAWC. The rat brain in stereotaxic coordinates. Fourth ed. San Diego: Academic Press; 1998.

Peng CC, Sun HT, Lin IP, Kuo PC, Li JC. The functional property of royal jelly 10-hydroxy-2-decenoic acid as a melanogenesis inhibitor. BMC Complement Altern Med. 2017;17:392.

Pyo JO, Yoo SM, Ahn HH, Nah J, Hong SH, Kam TI, et al. Overexpression of Atg5 in mice activates autophagy and extends lifespan. Nat Commun. 2013;4:2300.

Qiu W, Chen X, Tian Y, Wu D, Du M, Wang S. Protection against oxidative stress and anti-aging effect in Drosophila of royal jelly-collagen peptide. Food Chem Toxicol. 2020;135: 110881.

Rodriguez-Arribas M, Pizarro-Estrella E, Gomez-Sanchez R, Yakhine-Diop SM, Gragera-Hidalgo A, Cristo A, et al. IFDOTMETER: a new software application for automated immunofluorescence analysis. J Lab Autom. 2016;21:246-59.

Rubinsztein DC, Codogno P, Levine B. Autophagy modulation as a potential therapeutic target for diverse diseases. Nat Rev Drug Discov. 2012;11:709-30.

Ryu D, Mouchiroud L, Andreux PA, Katsyuba E, Moullan N, Nicolet-Dit-Felix AA, et al. Urolithin A induces mitophagy and prolongs lifespan in $\mathrm{C}$. elegans and increases muscle function in rodents. Nat Med. 2016;22:879-88.

Sarbassov DD, Guertin DA, Ali SM, Sabatini DM. Phosphorylation and regulation of $\mathrm{Akt} / \mathrm{PKB}$ by the rictormTOR complex. Science. 2005;307:1098-101.

Sarkar S, Davies JE, Huang Z, Tunnacliffe A, Rubinsztein DC. Trehalose, a novel mTOR-independent autophagy enhancer, accelerates the clearance of mutant huntingtin and alphasynuclein. J Biol Chem. 2007;282:5641-52.

Shakeri A, Cicero AFG, Panahi Y, Mohajeri M, Sahebkar A. Curcumin: a naturally occurring autophagy modulator. J Cell Physiol. 2019;234:5643-54.

Sheng Q, Xiao X, Prasadan K, Chen C, Ming Y, Fusco J, et al. Autophagy protects pancreatic beta cell mass and function in the setting of a high-fat and high-glucose diet. Sci Rep. 2017;7:16348.

Spannhoff A, Kim YK, Raynal NJ, Gharibyan V, Su MB, Zhou $\mathrm{YY}$, et al. Histone deacetylase inhibitor activity in royal jelly might facilitate caste switching in bees. EMBO Rep. 2011;12:238-43.

Sugiyama T, Takahashi K, Mori H. Royal jelly acid, 10-hydroxytrans-2-decenoic acid, as a modulator of the innate immune responses. Endocr Metab Immune Disord Drug Targets. 2012;12:368-76. 
Toth ML, Sigmond T, Borsos E, Barna J, Erdelyi P, Takacs-Vellai $\mathrm{K}$, et al. Longevity pathways converge on autophagy genes to regulate life span in Caenorhabditis elegans. Autophagy. 2008;4:330-8.

Vicinanza M, Korolchuk VI, Ashkenazi A, Puri C, Menzies FM, Clarke JH, et al. PI(5)P regulates autophagosome biogenesis. Mol Cell. 2015;57:219-34.

Wan DC, Morgan SL, Spencley AL, Mariano N, Chang EY, Shankar $\mathrm{G}$, et al. Honey bee Royalactin unlocks conserved pluripotency pathway in mammals. Nat Commun. 2018;9:5078.

Watadani R, Kotoh J, Sasaki D, Someya A, Matsumoto K, Maeda A. 10-Hydroxy-2-decenoic acid, a natural product, improves hyperglycemia and insulin resistance in obese/diabetic KKAy mice, but does not prevent obesity. J Vet Med Sci. 2017;79:1596-602.

Weiser MJ, Grimshaw V, Wynalda KM, Mohajeri MH, Butt CM. Long-term administration of queen bee acid (QBA) to rodents reduces anxiety-like behavior, promotes neuronal health and improves body composition. Nutrients. 2017;10.

Xin XX, Chen Y, Chen D, Xiao F, Parnell LD, Zhao J, et al. Supplementation with major Royal-Jelly proteins increases lifespan, feeding, and fecundity in Drosophila. J Agric Food Chem. 2016;64:5803-12.

Yakhine-Diop SM, Martinez-Chacon G, Gonzalez-Polo RA, Fuentes JM, Niso-Santano M. Fluorescent FYVE chimeras to quantify PtdIns3P synthesis during autophagy. Methods Enzymol. 2017;587:257-69.
Yang XY, Yang DS, Wei Z, Wang JM, Li CY, Hui Y, et al. 10Hydroxy-2-decenoic acid from Royal jelly: a potential medicine for RA. J Ethnopharmacol. 2010;128:314-21.

Yang A, Zhou M, Zhang L, Xie G, Chen H, Liu Z, et al. Influence of royal jelly on the reproductive function of puberty male rats. Food Chem Toxicol. 2012;50:1834-40.

Yang YC, Chou WM, Widowati DA, Lin IP, Peng CC. 10hydroxy-2-decenoic acid of royal jelly exhibits bactericide and anti-inflammatory activity in human colon cancer cells. BMC Complement Altern Med. 2018;18:202.

You M, Miao Z, Pan Y, Hu F. Trans-10-hydroxy-2-decenoic acid alleviates LPS-induced blood-brain barrier dysfunction by activating the AMPK/PI3K/AKT pathway. Eur J Pharmacol. 2019a;865:172736.

You M, Miao Z, Tian J, Hu F. Trans-10-hydroxy-2-decenoic acid protects against LPS-induced neuroinflammation through FOXO1-mediated activation of autophagy. Eur J Nutr. 2019b;59:2875-92.

Zhang L, Yu J, Pan H, Hu P, Hao Y, Cai W, et al. Small molecule regulators of autophagy identified by an image-based highthroughput screen. Proc Natl Acad Sci U S A. 2007;104: 19023-8.

Publisher's note Springer Nature remains neutral with regard to jurisdictional claims in published maps and institutional affiliations. 